\title{
Parallel Memory-Efficient Adaptive Mesh Refinement on Structured Triangular Meshes with Billions of Grid Cells
}

\author{
OLIVER MEISTER, KAVEH RAHNEMA, and MICHAEL BADER,
}

Department of Informatics, Technische Universität München

\begin{abstract}
We present $\operatorname{sam}(\mathrm{oa})^{2}$, a software package for a dynamically adaptive, parallel solution of $2 \mathrm{D}$ partial differential equations on triangular grids created via newest vertex bisection. An element order imposed by the Sierpinski space-filling curve provides an algorithm for grid generation, refinement, and traversal that is inherently memory efficient. Based purely on stack and stream data structures, it completely avoids random memory access. Using an element-oriented data view suitable for local operators, concrete simulation scenarios are implemented based on control loops and event hooks, which hide the complexity of the underlying traversal scheme. Two case studies are presented: two-phase flow in heterogeneous porous media and tsunami wave propagation, demonstrated on the Tohoku tsunami 2011 in Japan.

$\operatorname{sam}(\text { oa })^{2}$ features hybrid MPI+OpenMP parallelization based on the Sierpinski order induced on the elements. Sections defined by contiguous grid cells define atomic tasks for OpenMP work sharing and stealing, as well as for migration of grid cells between MPI processes. Using optimized communication and load balancing algorithms, sam(oa $)^{2}$ achieves $88 \%$ strong scaling efficiency from 16 to 512 cores and $92 \%$ efficiency in a weak scaling test on 8,192 cores with 10 billion elements-all tests including adaptive mesh refinement and load balancing in each time step.
\end{abstract}

CCS Concepts: $\bullet$ Computing methodologies $\rightarrow$ Massively parallel and high-performance simulations

Additional Key Words and Phrases: Parallel adaptive mesh refinement, space-filling curve, cache efficient, memory efficient, hybrid parallelization, porous media flow, tsunami wave propagation

ACM Reference Format:

Oliver Meister, Kaveh Rahnema, and Michael Bader. 2016. Parallel memory-efficient adaptive mesh refinement on structured triangular meshes with billions of grid cells. ACM Trans. Math. Softw. 43, 3, Article 19 (September 2016), 27 pages.

DOI: http://dx.doi.org/10.1145/2947668

\section{INTRODUCTION}

Due to the current hardware trend toward smaller, cheaper computation units with less memory per core, memory- and cache-aware algorithms have become increasingly important. Similarly, simulation software needs to cope with the growing number of cores on modern CPUs. With the presented package sam $(\mathrm{oa})^{2}$ (Space-filling curves and Adaptive Meshes for Oceanic And Other Applications, we provide a dynamically adaptive solver for 2D Partial Differential Equations (PDEs) that combines memory efficiency with hybrid parallelization and flexibility with respect to element-oriented discretization approaches.

Authors' addresses: O. Meister, K. Rahnema, and M. Bader, Department of Informatics, Technische Universität München; Boltzmannstr. 3, 85748 Garching b. M., Germany; emails: oliver.meister@tum.de, kaveh.rahnema@mytum.de, bader@in.tum.de.

Permission to make digital or hard copies of all or part of this work for personal or classroom use is granted without fee provided that copies are not made or distributed for profit or commercial advantage and that copies bear this notice and the full citation on the first page. Copyrights for components of this work owned by others than the author(s) must be honored. Abstracting with credit is permitted. To copy otherwise, or republish, to post on servers or to redistribute to lists, requires prior specific permission and/or a fee. Request permissions from Permissions@acm.org.

2016 Copyright is held by the owner/author(s). Publication rights licensed to ACM.

0098-3500/2016/09-ART19 $\$ 15.00$

DOI: http://dx.doi.org/10.1145/2947668 
Memory efficiency requires Adaptive Mesh Refinement (AMR) in order to invest Degrees of Freedom (DoFs) where they contribute most to the solution. To solve wave propagation problems, for example, refinement along wavefronts, which are typically of lower (fractal) dimension than the domain [Popinet 2011], restricts the finest grid resolution to only fcells. In the case of tsunami simulation, the domain may extend over thousands of kilometers, but interesting features on the coastline may only be a few meters wide [LeVeque et al. 2011]. A regular grid that resolves this difference of six orders of magnitude in resolution would be prohibitively expensive. Consequently, we focus on cell-wise adaptivity, in contrast to patch-based approaches [Burstedde et al. 2013; Weinzierl et al. 2013], and target dynamical refinement in each time step.

We strive to support Finite Element (FE), Finite Volume (FV), and Discontinuous Galerkin (DG) discretizations without changes to the program core (grid refinement, grid traversal schemes, parallelization, etc.). Hence, $\left(\operatorname{sam}(\mathrm{oa})^{2}\right)$ requires an abstraction of the grid traversal process by means of an event-based interface that calls applicationspecific kernels provided by the developer or third-party sources. Cache-friendly memory access is ensured with a strictly element-oriented event paradigm. This design allows application developers to implement efficient element-local operators on the grid without knowledge of underlying traversal processes.

Finally, we consider hybrid parallelization as imperative to decrease memory per core and minimize the time to solution, exploiting shared and distributed-memory architectures. Not only does this reduce the execution time of scenarios, but also it allows bigger scenarios with deeper refinement, such as the described tsunami scenario. Dynamic load balancing (after each time step, if desired) must also be taken care of, due to strongly varying refinement and coarsening of the solution and kernels such as flux solvers with spatially and temporally inhomogeneous execution times.

$\operatorname{sam}(\mathrm{oa})^{2}$ tackles memory efficiency via the concept of Sierpinski traversals, which we recapitulate in Section 1: based on a sequential order induced on the triangular grid cells via a Sierpinski Space-Filling Curve (SFC), element-oriented, matrix-free algorithms that require only inherently memory-efficient stack and stream data structures are used for the dynamically adaptive solution of PDEs. In Section 2, we describe a kernel-oriented design in $\operatorname{sam}(\mathrm{oa})^{2}$ that combines features from FE, FV, and DG frameworks with frameworks for parallel adaptive mesh refinement. It extends our previous design presented in Meister et al. [2012] to better support FV-type simulations. The prime contribution of this article is the hybrid parallelization approach presented in Section 3. Parallelization and load balancing are based on subpartitions defined via the Sierpinski order on the grid cells. These so-called Sierpinski sections are treated as atomic work units for distributed-memory parallelism, while the partitioning and load-balancing problem is solved in a shared-memory setting.

We especially target problems of low or medium arithmetic intensity with a performance that is bound or at least noticeably influenced by memory bandwidth. In such scenarios, performance penalties due to complicated data structures for adaptive models, as well as overhead due to adaptive refinement and load balancing, are much more pronounced compared to high-order discretizations or problems with expensive compute-bound solvers. Two case studies, presented in Section 4, reflect this focus: two-phase flow in heterogeneous porous media and tsunami wave propagation. The first example features a semi-implicit time-stepping method on a dual-grid discretization. A new fused Conjugate Gradients (CG) solver is applied, optimized for low memory footprint, for the solution of a pressure equation in each time step. The linear system is comparably cheap to solve; thus, performance is strongly memory bound. The second problem uses an explicit Finite Volume method with moderately expensive Riemann solvers for the underlying Shallow Water Equations. In this fully explicit method, adaptivity and parallelization costs are expected to have a strong impact on 


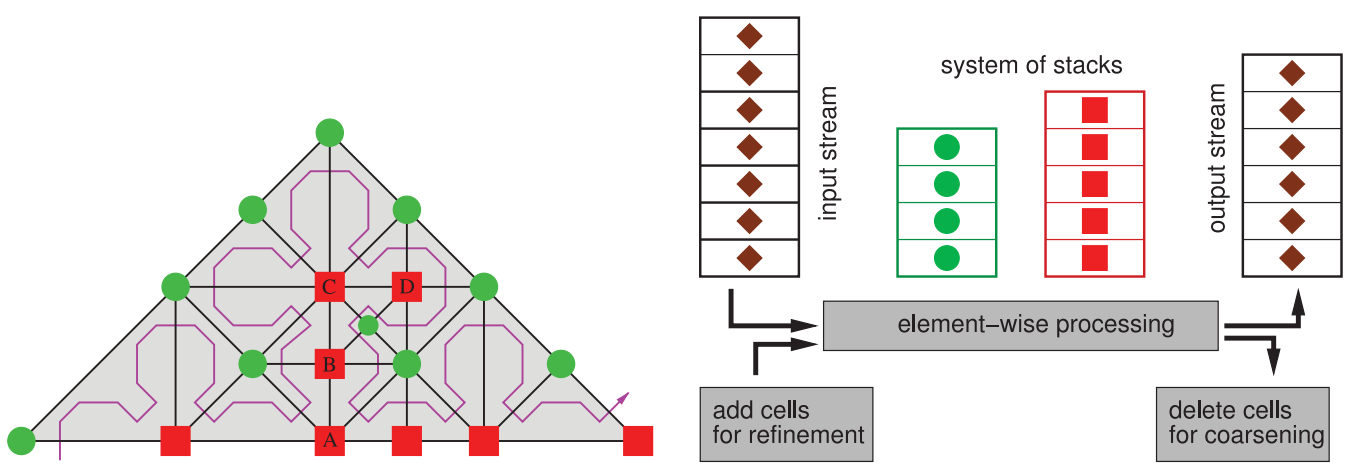

Fig. 1. Structured adaptive grid using newest vertex bisection (left image) and motivation of the stack \& stream approach (right image). During a grid traversal in Sierpinski order (purple curve), vertices on the left (green) side and on the right (red boxes) side of the curve (see vertices A-D) are accessed in order when they are touched by an element for the first time and accessed in reverse order when they are touched for the last time, which suggests a stack-compatible access pattern. An automaton (right image) implements the full mechanism: it reads edges and vertices from an input stream on their first touch and pushes them to red/green stacks. Intermediate touches (e.g., of vertex B, which is adjacent to five elements) require only access to the stacks. After their last touches, the automaton pushes edges and vertices to the output stream. Image source: Bader et al. [2010].

performance. For both scenarios, we evaluate scalability and performance for sharedand distributed-memory parallelism in Section 5.

\section{HISTORY OF SIERPINSKI TRAVERSALS FOR DYNAMICALLY ADAPTIVE PDE SOLVERS}

In this article, we recapitulate previous work [Bader et al. 2008, 2010] on dynamically adaptive mesh traversals based on the Sierpinski curve and how they are used to efficiently solve specific simple PDEs and systems of PDEs.

Triangular grids are created and adaptively refined via newest vertex bisection [Mitchell 1991], which together with a Sierpinski SFC order on the grid cells (see Figure 1, left image) allows a linearized storage of a fully adaptive grid with very low memory requirements. PDE solvers are formulated in an element-oriented, matrix-free way, such that an assembly of global system matrices is avoided. The Sierpinski order (purple line in Figure 1, left image) further induces a stack property on node- and edge-located unknowns, such that during grid traversals repeated accesses to these unknowns are organized via stream and stack data structures. In each grid traversal, all unknowns are initially located on an input stream (rhombi on the left in Figure 1, right image). When an element accesses an unknown for the first time (see vertices A-D in Figure 1, left image), it is read from the input stream, processed, and pushed either to the green or the red stack (red squares in Figure 1, right image), depending on whether it is located left or right of the curve. On all accesses by further elements, the unknown is popped from the stack. Similarly, it is pushed to the stack after all accesses except for the last, after which the unknown is written to the output stream (rhombi on the right in Figure 1, right image).

Access rules to stacks and streams are deterministic and lead to nested recursive traversal algorithms that are derived alongside the recursive description of the Sierpinski curve; a detailed description of the access rules is given in Bader et al. [2010]. One major gain from such a framework is the avoidance of redundant data, such as separation of "old" and "new" unknowns. Instead, temporary variables on the stack store intermediate values with no persistent memory footprint. The strict traversaloriented access policy controls read and write access to unknowns and issues creation and deletion of temporary unknowns and updates to persistent variables. 


\subsection{Efficient Traversal on Triangle Strips}

If processing is restricted to fine-grid cells, we apply a nonrecursive, loop-based implementation (described in detail in Vigh [2012]), similar to the use of triangle strips in computer graphics [Pajarola 1998; Velho et al. 1999]. Both the recursive and the loop-based implementation exploit the inherent data locality provided by the spacefilling orders and lead to excellent cache efficiency of the approach. Explicit storage of a refinement tree is not required. While hierarchical algorithms such as geometric multigrid methods benefit from the recursive approach [Bader et al. 2008], the loop-based implementation was demonstrated to achieve better memory performance [Bader et al. 2011]. On the other hand, the strong integration of data structures for grids and unknowns (which is rather typical for Cartesian or block-structured grids) and the traversal-oriented approach makes the implementation of applications difficult and more tedious than for classical approaches for unstructured grids, where grid representation and numbering of unknowns are typically well separated. Hence, our goal was to provide a suitable "front end" [Meister et al. 2012] for easier implementation of especially element-oriented PDE solvers, which, however, retains the superior memory access properties and resulting performance advantages of structured grids also for computations on fully adaptive meshes; see the kernel design in Section 2.

\subsection{Parallelization with Sierpinski Space-Filling Curves}

The stack\&stream approach imposes a sequential workflow due to the traversal order required for stack and stream consistency, which prevents simple loop-based paradigms for shared-memory parallelism. However, established approaches for parallelization with SFCs may be adopted. These exploit that space-filling curves provide good heuristics to approximately solve the NP-hard problem of load balancing on grids subject to a minimization of the communication constraint. A standard approach to generate balanced partitions is to sequentialize all grid cells based on the Sierpinski order and then select contiguous intervals in the SFC order as partitions. Stack-based data exchange can then be implemented via message passing: data at partition boundaries is stored on separate stacks, which act as a dynamic ghost layer. The Hölder-continuity of space-filling curves [Zumbusch 2001] ensures reasonably small partition boundaries. Further information on parallelization of structured adaptive triangular grids is found in the work by Behrens and Zimmermann [2000] and Zumbusch [2002]. The REFTREE algorithm [Mitchell 2007] exploits the tree structure induced by the bisection-based refinement to compute balanced partitions on Sierpinski-ordered triangular grids. In Schreiber et al. [2012], an analogous idea is applied to the Sierpinski-traversal concept and extended toward a massive split-and-join approach that schedules subtree partitions to available compute resources in a dynamic fashion. For adaptive Cartesian grids (quadtrees, octrees, etc.), SFC- and tree-based dynamic load balancing is successfully applied in p4est [Burstedde et al. 2011] or Peano [Weinzierl and Mehl 2011]; for example, in Section 3, we introduce a NUMA-aware approach for hybrid shared- and distributed-memory parallelization, based on so-called Sierpinski sections.

\section{SAM(OA) ${ }^{2}$ : A KERNEL-BASED DESIGN TO IMPLEMENT APPLICATIONS}

A framework to implement applications in sam $(\text { oa })^{2}$ especially needs to hide the complexity of the stack\&stream approach while providing flexibility for multiple discretization methods, offering refinement and coarsening methods, and still being fairly easy to use. $\operatorname{sam}(\mathrm{oa})^{2}$ thus defines multiple abstraction layers, where each layer only provides the most necessary data.

The main concepts were inspired by similar frameworks: Peano [Bungartz et al. 2010] shares the stack\&stream approach with sam $(\text { oa })^{2}$ but is based on tree-structured 


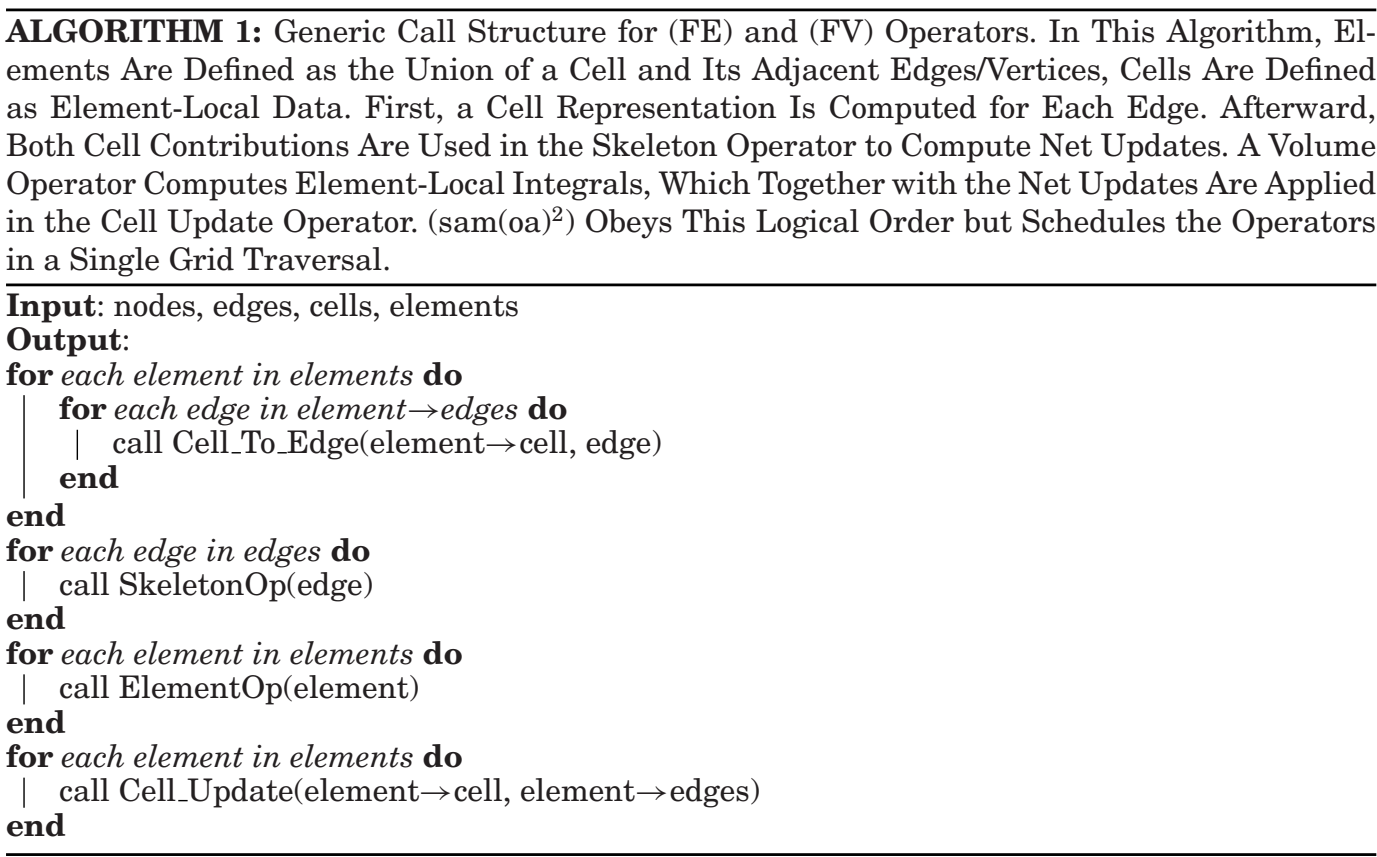

adaptive Cartesian grids (similar to octrees) and the Peano SFC. It provides a simple yet powerful interface composed of grid events, or hooks, called during a traversal, for example, when first and last accesses (touches) of vertices occur. These hooks may be implemented for concrete applications and can be combined in multiple traversals to yield complex algorithms. $\operatorname{sam}(\text { oa })^{2}$ relies on a similar hook-based interface that uses First- and Last-Touch operators for implementation of per-vertex or per-edge operations. These allow initialization and updates of unknown vectors.

PDELab in DUNE (Distributed and Unified Numerics Environment [Bastian et al. 2008] (DUNE)) introduces the concept of volume and skeleton kernels having a residual-oriented description of operators in mind that is based on weak forms of PDEs. The concept of skeleton and element operators in PDELab is also used in $\operatorname{sam}(\mathrm{oa})^{2}$, as it provides a powerful tool for implementation of many FE, FV, and DG methods.

Dolphin [Logg and Wells 2010] describes applications in a domain-specific language, the Unified Form Language [Alnæs et al. 2014] (UFL), and provides automated discretization for FE-type problems. AMR is provided based on longest edge bisection and automatic error indicator generation for refinement and coarsening. The main input was the idea of formulating a generalized refinement/coarsening strategy based on local operators. The refinement and coarsening operators in $\operatorname{sam}(\mathrm{oa})^{2}$ reflect that idea.

To support FE, FV, and DG models in an AMR context, $\operatorname{sam}(\text { oa })^{2}$ defines two distinct types of traversals. The static grid traversal allows one to execute element-based kernels that may change any data but cannot modify the topology of the grid. For AMR, a dedicated adaptive grid traversal with a similar generic interface is added that encapsulates explicit data transfer between two distinct grids. Both concepts for static and adaptive traversal are presented in this section.

\subsection{Kernel Concept for Static Grid Traversals}

Algorithm 1 displays the logical execution order of generic FE and FV operators in $\operatorname{sam}(\mathrm{oa})^{2}$ : In the first loop, a cell-to-edge operator computes an edge representation 


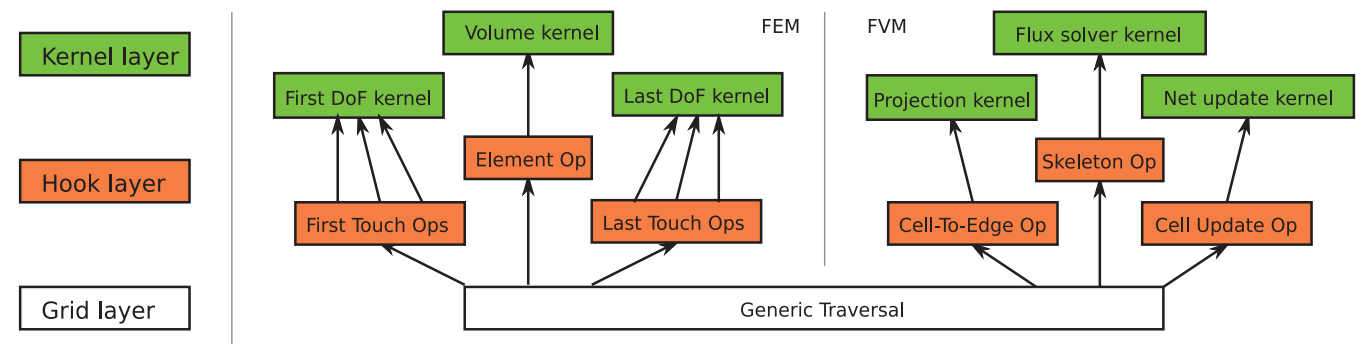

Fig. 2. Layer concept: Event-based call structure of a grid traversal. The grid layer is invisible to the user and raises events in the hook layer. Here, data in the grid is accessed and passed to application-specific kernels. First touch/last touch operators for cells, edges, and nodes typically call the same DoF kernel, which may initialize a vector or perform an update (hence three arrows for cells, edges, and vertices each). Kernels are implemented by either the user or external functions (e.g., from libraries or other packages).

of the cell-local Degree of Freedoms (DoFs) for all element edges. In the case of cellcentered FVs, only a simple copy of the cell data to the edge is required. In a DG context, the operator would project a cell-local state function to each edge. In the following loop, a skeleton operator computes an update from both edge representations, for example, by quadrature of an approximate Riemann solution on each edge to compute net updates for both adjacent cells. An element operator then executes element-local operations, such as the product of the element stiffness matrix and quantity vector. Finally, the cell update operator applies the update to the adjacent cells. Internally, it accumulates the computed net updates from all adjacent edges and applies them to cell-local quantities.

The stack\&stream approach allows out-of-order execution of the loops in Algorithm 1, as long as data dependency is preserved. The skeleton operator can be executed on an edge as soon as the cell-to-edge operator has been called for both neighbor cells. Similarly, the cell update operator can be called as soon as the skeleton operator has been executed for all three adjacent edges. With this idea in mind, sam(oa $)^{2}$ implements Algorithm 1 as a pipelined scheme that requires only a single grid traversal to execute all FV operators while preserving data dependency. Note that the described interface supports only FE and FV methods with compact stencils. Bigger stencils in each grid iteration would effectively require random access to elements and contradict the stack\&stream system.

$\operatorname{sam}(\mathrm{oa})^{2}$ supports and implements this kernel-based application interface based on a layer concept (illustrated in Figure 2) for generic traversals on static grids. It combines our previous FE-oriented interface [Meister et al. 2012] by the components necessary for the FV-oriented kernels. The fundamental grid layer holds the program core and contains the implementation of all grid- and data-related base functionality, including all stack\&stream-based memory transfers. The grid layer is completely hidden from an application developer (referred to as user from here on). Instead, it raises grid events to the hook layer, which reflects the structure of Algorithm 1. The stack\&stream system enforces a set of data visibility rules: an element may access only its associated cell, edge, and vertex data, and each edge or vertex may only access its own data. All operators on this layer are designed to adhere to these rules. Unknowns or matrices are read from or written to the grid and may be passed as arguments to applicationspecific kernels in the kernel layer. Kernels can either be external functions (e.g., from libraries) or are implemented by the user. For example, a Volume kernel performs a computation on element-local data (such as a matrix-vector product with the local system matrix), and a DoF kernel operates on single DoFs (e.g., to apply a vector update). This additional abstraction layer decouples the grid from the application in an adapter pattern, which serves two purposes: it allows further abstraction to better 


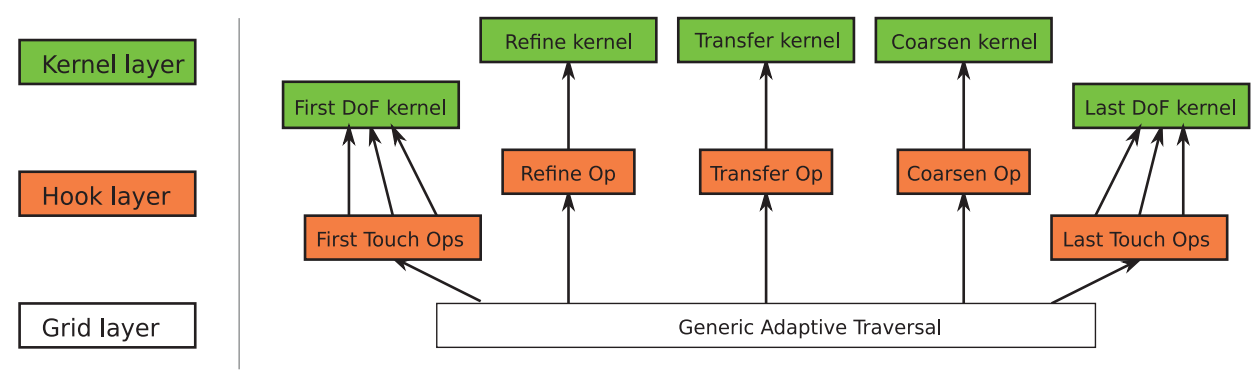

Fig. 3. Adaptive layer concept: again, the grid layer hides traversal from the user, who implements the hook layer and may call external kernels in the kernel layer or implement his or her own kernels for refinement, transfer, and coarsening of grid cells. Refinement and coarsening kernels depend on the choice of basis functions and may be external or access external data.

structure the implementation of model variants (varying approximation order, source terms, etc.) and it allows wrapping of kernels from other simulation software packages.

Kernels may be combined by execution of multiple operators in the hook layer in order to formulate complex algorithms. Section 4.1 demonstrates application of the FE interface to implement a CG solver and a node-centered FV method. The FV interface is used in Section 4.2 to implement a time-stepping method with external flux solvers.

\subsection{Kernel Concept for Adaptive Mesh Refinement}

The AMR functionality in $\operatorname{sam}(\mathrm{oa})^{2}$ operates on a cell level: each cell may be marked for refinement or coarsening, for example, by setting a flag subject to an error indicator implemented in an element kernel. Then, dedicated traversals ensure grid conformity (i.e., avoid hanging nodes) and perform refinement and coarsening of all grid cells, together with the corresponding interpolation operations on the DoFs. In that way, the kernel design provides fine-grained control over cell-oriented local refinement to minimize memory and computation overhead caused by unnecessary grid refinement.

We enforce the layer approach also for AMR and interpolation/restriction during dynamically adaptive simulations. A generic adaptive traversal for grid refinement and coarsening is provided, where the user specifies first- and last-touch operators, as well as refinement, coarsening, and transfer operators on the hook layer (see Figure 3). The respective operators may be applied for single and two-step bisection of an element. Due to the option to implement refinement/coarsening kernels, complicated interpolation and restriction operations can be realized in $\operatorname{sam}(\text { oa })^{2}$; in Section 4.1 , we describe the mass-conserving interpolation for a porous-media-flow problem as an example.

In static grid traversals, the input and output streams for node, edge, and cell data, respectively, have a fixed total size and are thus stored as a single combined array data structure, which leads to improved performance compared to using two separate arrays or a list- or vector-based implementation. For the adaptive traversal, a single array does not suffice, as some array entries must be removed during coarsening and new elements must be inserted during refinement. Here, we temporarily allocate a second array and use it as the output stream while the old array is used as the input stream. While this doubles the amount of allocated memory temporarily, it proved to achieve better overall performance than alternative list- or vector-based implementations.

\section{PARALLELIZATION USING SIERPINSKI SECTIONS}

As $\operatorname{sam}(\text { oa })^{2}$ is designed to work on triangle strips as a data structure, it partitions the grid by cuts of the SFC. We call these partitions SFC sections and treat them as independent, atomic parallel units. A section consists of a sequence of contiguous, 

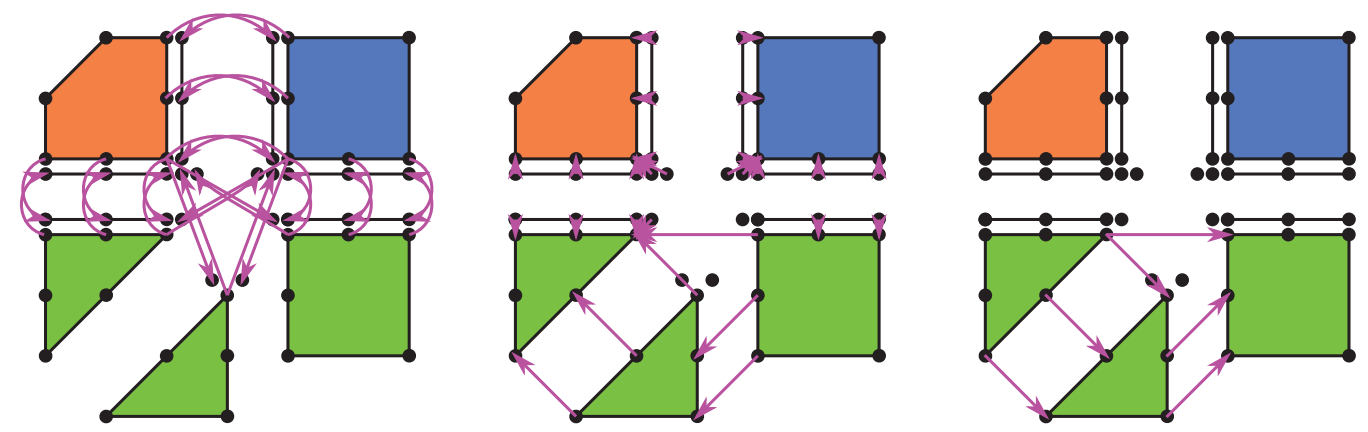

Fig. 4. Three-step hybrid OpenMP/MPI communication model for vertex data (similar for edge data): each section sends its process boundary vertices to the ghost vertices of all neighbor processes and receives their process vertices in its own ghost vertices (left image, arrows between sections with distinct colors). Next, vertex data from process-local neighbor sections and ghost vertices is accumulated in a designated owner section for each vertex (middle image). Finally, the data is distributed back from the owner section to the other process-local sections (right).

edge-connected grid cells as defined by the Sierpinski order. With the cells, adjacent edges and vertices are stored in their associated streams and stacks. Each section has its own communication structure that stores communication partners, that is, other sections, located on the same MPI rank or on remote MPI ranks.

While each section must be traversed in order and cannot be executed in parallel, multiple sections may be processed in parallel and out of order. Both in distributed and shared memory, sections are scheduled as tasks in a coarse-grained manner and we allow multiple sections to be assigned to a single thread or process.

\subsection{Communication Over Stacks}

Communication of boundary data is performed via special boundary stacks, which are used on the partition boundary instead of regular stacks. These stacks provide a persistent memory location for all communication data, and are thus accessible by neighbor partitions. A run-length encoded list (as introduced in Schreiber et al. [2012]) of neighbors then exploits the SFC stack property: a strictly increasing monotonic numbering of partitions results in a strictly decreasing monotonic access to neighbor partitions. Communication between partitions is therefore not fragmented and the runlength encoded lists efficiently compress the neighbor information, thus contributing to memory efficiency of $\operatorname{sam}(\mathrm{oa})^{2}$.

Shared Memory Communication. For shared memory communication, each section may directly access its own and neighbor boundary stacks. This avoids data copying, but as a tradeoff, data exchange must be divided into an accumulation phase and a broadcast phase in order to rule out race conditions. Figure 4 demonstrates this idea for vertex exchange between the green sections. Each vertex has a designated owner section that is responsible for accumulating contributions from neighbor sections. Once all contributions are collected (verified by an OpenMP barrier), the result is distributed back to all neighbors.

Distributed-Memory Communication. To implement overlapping of communication and computation, we require separate send and receive buffers for MPI communication. The boundary stack acts as a send buffer, and a copy of the boundary stack is used as a receive buffer. In Figure 4, MPI vertex communication between sections with distinct colors is sketched. Each vertex is sent asynchronously to each MPI neighbor 


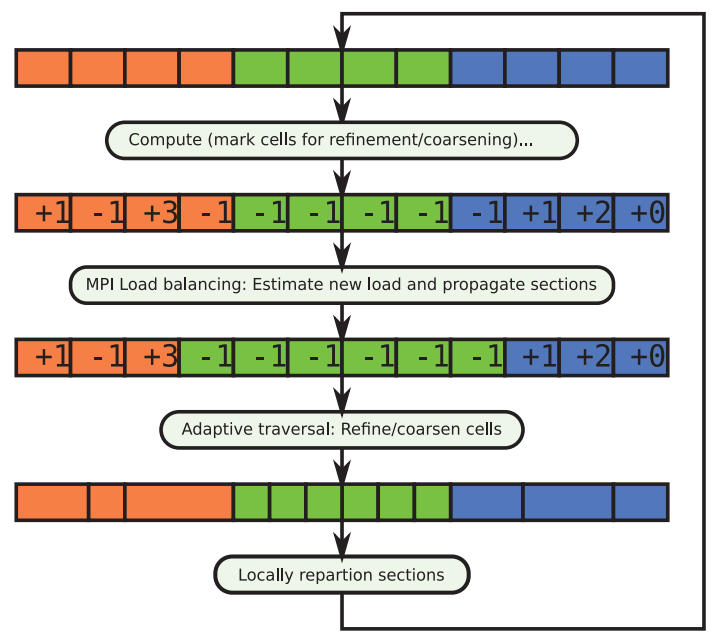

Fig. 5. Hybrid MPI/OpenMP load-balancing assignment in four steps: (1) Starting with a balanced grid with sections of uniform count and size, mark each section with the number of additional cells requested by refinement and coarsening indicators. (2) Distribute sections over MPI ranks to achieve approximately uniform load per MPI rank. (3) Locally refine and coarsen sections. (4) Locally repartition sections to uniform count and size again.

and accumulated locally in the stack of the owner section once all data is received. As in shared memory, the accumulated data is sent to all local sections afterward.

\subsection{Load Balancing}

Figure 5 shows a four-step algorithm for hybrid MPI/OpenMP load balancing. In step 1, starting with sections of roughly uniform count and size on each core, an error indicator marks cells for refinement and coarsening during numerical traversals. Each section counts the cells that are inserted and removed and the respective gain or loss of load due to adaptive refinement and coarsening. In step 2 , this information is used to perform coarse MPI load balancing that distributes sections to MPI processes such that each MPI rank has roughly the same load-this step is omitted in a pure OpenMP parallelization. In step 3, the grid is adaptively refined and coarsened, now leading to uniform load per MPI process. However, the sections may still have different sizes, so load balance among OpenMP threads is not yet achieved. Step 4 therefore repartitions the process-local sections to uniform size. At this point, all sections have roughly uniform size again and can be assigned to threads for execution.

In the following, the load balancing and repartitioning steps are described in detail.

Shared Memory Load Balancing. OpenMP provides two approaches for load balancing between threads: work sharing and work stealing. Work sharing is supported in $\operatorname{sam}(\mathrm{oa})^{2}$ by NUMA-aware static assignment of sections to threads. As all sections should have a similar cell count after remeshing, sam $(\text { oa })^{2}$ assigns the same number of sections to each core and takes care that sections are assigned only to the NUMA domains they have been created on.

This approach works well for scenarios with homogeneous load per cell. Complex kernels may contain branches or early-exit conditions, however, and are inhomogeneous in space and time. $\operatorname{sam}(\text { oa })^{2}$ supports these scenarios by optionally activating OpenMP task constructs around each section traversal. Hence, each section is, in a NUMAaware fashion, preferably processed by the thread it is assigned to, but may be stolen by different threads if they finish their work before the owner thread claims its section. 
The more sections a core owns, the better is the load balance. A tradeoff exists though, since sections generate overhead due to additional communication. As can be seen in the performance analysis in Section 5.2.2, the ideal amount of sections per core depends on hardware, problem size, and level of parallelism, but is usually between two and 16 .

Repartitioning in Shared Memory. As seen in Figure 5, sam(oa) ${ }^{2}$ relies on a mechanism to locally repartition sections for distribution of load among threads and processes-ideally into arbitrary count and size. To avoid an extra, inherently expensive grid traversal, repartitioning is combined with adaptive refinement into a single step that requires only a single traversal.

Thread-parallel execution of this adaptive traversal is challenging, as multiple destination sections may require grid data from the same source sections. Threaded creation of destination sections consequently causes concurrent access to streams and concurrent access to stacks of the old grid. Concurrent access to stacks is handled by employing distinct stacks for each thread. Concurrent access to streams may happen but is restricted to concurrent read access and thus cannot cause race conditions. It may degrade performance, however, due to resource contention. If the number of sections per thread is set to at least two, the probability of concurrent stream access is reduced a lot and most read accesses will be exclusive again. For MPI load balancing, subpartitioning to at least two sections per thread is necessary anyway.

Distributed-Memory Load Balancing. sam(oa $)^{2}$ applies a strictly 1D load-balancing scheme that preserves the Sierpinski order. In regular operation, partitions grow or shrink through migration of sections from MPI ranks that are neighbors in the Sierpinski order. In addition, sections may be split or contiguous sections may be merged. As an important feature, varying load per grid elements may be considered.

The load-balancing problem in $\operatorname{sam}(0 a)^{2}$ is therefore described by the problem of finding a function $f:\{1, \ldots, n\} \rightarrow\{1, \ldots, c\}$ that maps $n \in \mathbb{N}$ sections to $c \in \mathbb{N}$ target cores. Given the load $l_{j}>0$ for each section $j \in\{1, \ldots, n\}$, with $\sum_{j=1}^{n} l_{j}=1$, $f$ has to fulfill two conditions: it must be monotonic to preserve the order of sections and it should minimize the maximum load per core $\max _{i \in\{1, \ldots, c\}} \sum_{j \in f^{-1}(i)} l_{j}$ to achieve the best load balance. The problem of finding such a function $f$ is known as chains-on-chains partitioning (CCP) and has been investigated extensively, for example, in Pinar and Aykanat [2004]. Unfortunately, no scalable algorithms are known to find an optimal solution. An approximation to be used in massively parallel settings is suggested by Pinar et al. as

$$
\tilde{f}(j):=\left\lfloor\left(\frac{l_{j}}{2}+\sum_{k=1}^{j-1} l_{k}\right) \cdot c\right\rfloor \quad \text { for } j=1, \ldots, n .
$$

This function divides the total load, consisting of an ordered set of all sections, into parts of equal size and assigns each part to a core. The load of each section is ideally assigned to a single core. If a section is assigned to multiple cores, the center of the section, which splits its load exactly in half, decides the target. The load imbalance caused by this approximation is limited by the maximum load per section and thus improves with the number of sections per core.

In our implementation, the total load is obtained from an MPI_Allreduce and used to compute the relative load $l_{j}$ for each section. On each section, $\tilde{f}$ is now evaluated according to Equation (1) (requiring an MPI_Scan to compute all sums $\sum_{k=1}^{j-1} l_{k}$ ) and the section is assigned to its new core. The set of cores given by evaluation of $\tilde{f}$ on all sections of a core are the destination cores. The respective algorithm leads to efficient and little data migration because a continuous 1D mapping of indices to cell coordinates 


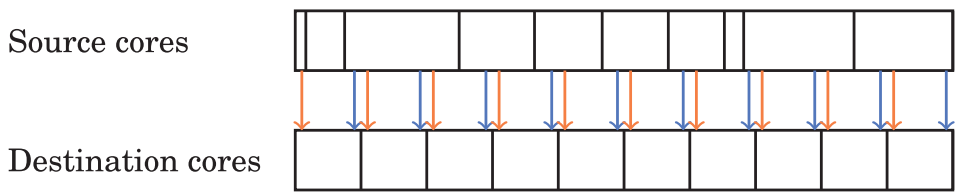

Load distribution

Fig. 6. CCP data distribution: Each core is represented by a bar, whose width equals the load of the core before (top) and after (bottom) load balancing. The source cores collectively compute the exclusive prefix sum over the process load. Then, each core finds its first destination core via Equation (1). The last destination rank for each core is found by using the inclusive MPI prefix sum.

exists due to the SFC. Discontinuous mappings would lead to fractured data exchange and poor MPI performance. Each core also needs its set of source cores, that is, the set of cores that contain all incoming sections to be received. This set is obtained without collective operations, using identification messages by the source cores instead (compare Figure 6).

Two steps remain: update of communication structures (see Section 3.3) and load propagation (i.e., migration of sections). Load propagation is straightforward, as for each section in the source grid the destination core is known and vice versa. Thus, in a global propagation step, sections are sent directly to their destination and the algorithm is finished. Note that communication between processes with a large rank difference may cause latency issues. In Schloegel et al. [1998], it is suggested that iterative strategies that restrict migration to local process neighbors may avoid this problem. For frequent load balancing, changes in partitioning are often small, such that the overhead from propagation detours is smaller than the overhead caused by latency. Hence, we also implemented a local load distribution algorithm that allows migration only between processes with consecutive ranks. The resulting imbalance will be handled in load-balancing iterations of successive time steps. Both global and local propagation have been implemented in $\operatorname{sam}(\mathrm{oa})^{2}$ and are compared in Section 5.2.1.

\subsection{Setup of Communication Structures}

$\operatorname{sam}(\text { oa })^{2}$ exploits its capabilities for dynamically adaptive mesh refinement to incrementally set up the initial grid. Starting with a coarse grid that consists of only two triangles in a single section on a single core, the grid is successively refined and distributed among processes until the desired resolution has been reached everywhere. At this point, the grid is fully refined and load balance is ensured among all processes.

The same process is used to set up communication structures:

(1) Initially a single section exists on a single core.

(2) The grid is refined and split into multiple sections on each MPI process. For each process, all local communication structures are rebuilt, but communication among MPI processes is topologically unchanged.

(3) MPI load balancing is applied. Each migrating section informs all its neighbor sections of its new MPI rank.

(4) If the maximum grid depth is not obtained yet, go back to step 2.

For step 2, we developed a new algorithm that again exploits the stack\&stream property of the SFC. Assuming the whole grid is stored in a single section, then all section boundary vertices become inner vertices and will be pushed either to the red or to the green stack at some point. If a vertex is removed from the stack, it will either be pushed back immediately or it will be written to the output stream. Hence, a vertex must be fully processed if it was on the stack and the height of the stack has since 
decreased. Assuming we store the height of the stack for each vertex at the time it is pushed-its stack index-and split the grid into sections, we can now decide if the vertex is shared by other sections: two vertices $v$ and $w$ are identical if and only if they have the same stack index and there is no vertex $u$ with a lower stack index than $v$ and $w$ between them (in Sierpinski order). Thus, in order to find all neighbor sections, each section determines the smallest stack index for all its boundary vertices and then successively asks all neighbor sections (in Sierpinski order) for their smallest stack index, starting with itself. The algorithm stops if a section has a smaller index than the start index or if the domain boundary is hit. Four passes are necessary per section to find all neighbors: for the red and green stack in both the backward and forward direction.

In step 3, the communication structures do not have to be rebuilt locally; a simple traversal of the data structure is sufficient. The source rank and section index are updated to the values of the destination rank and section index. Afterward, this information is sent to all neighbor sections in order to inform them that they should update their destination rank and section index as well. In total, this step is as expensive as a regular communication phase.

Later, during execution of time steps, the same process (i.e., steps 2-4) is used to update communication structures after grid refinement and coarsening.

\section{APPLICATIONS}

With its element-based approach and focus on memory and cache efficiency, $\operatorname{sam}(\mathrm{oa})^{2}$ primarily targets memory-bound applications that require dynamically adaptive refinement. Two such scenarios are chosen here for evaluation of parallel performance. The first scenario simulates multiphase flow in porous media using an FE discretization. In each time step, pressure correction requires the solution of a Linear System of Equations (LSE), which is a classical example of an expensive task with low arithmetic intensity and typically memory-bound performance, as defined by the roofline model [Williams et al. 2009]. Phase saturation is discretized with an FV approach on a dual grid, which poses a challenge for AMR. To ensure mass conservation, interpolation and restriction require accumulation of element mass contributions, which is implemented via the AMR kernels in sam(oa $)^{2}$.

The second application discretizes the shallow water equations with an FV method in order to simulate tsunami wave propagation. The Tohoku tsunami from 2011 is used as an example scenario. We used versatile nonlinear Riemann solvers on the element interfaces to capture specific effects, such as inundation at coasts. Integration of these solvers is supported by the hook and kernel layer in $\operatorname{sam}(\mathrm{oa})^{2}$. The goal for both implementations was to express the numerical algorithms in a kernel formulation that reduces the number of grid traversals in order to minimize memory accesses.

\subsection{Two-Phase Porous Media Flow}

The first test case simulates multiphase flow on a heterogeneous porous medium with smooth permeability transitions and nonlinear flux following an approach by Chueh et al. [2010]:

$$
\begin{array}{cr}
0=\Phi S_{t}+\operatorname{div}(F(S) \mathbf{u}), & \operatorname{with} F(S)=\frac{\lambda_{w}(S)}{\lambda_{w}(S)+\lambda_{n}(S)}, \\
\mathbf{u}=-\left(\lambda_{w}(S)+\lambda_{n}(S)\right) K \nabla p, & \text { with } \lambda_{w}(S)=\frac{S^{2}}{\mu_{w}}, \lambda_{n}(S)=\frac{(1-S)^{2}}{\mu_{n}} .
\end{array}
$$

The system couples two unknowns, pressure $p$ and saturation $S$, and is solved by a two-step Implicit Pressure, Explicit Saturation (IMPES) scheme [Binning and Celia 
1999]. First, $p$ is determined by solving a Poisson equation:

$$
\nabla \cdot \mathbf{u}=\nabla \cdot\left(-\left(\lambda_{w}(S)+\lambda_{n}(S)\right) K \nabla p\right)=0 .
$$

Second, $S$ is computed explicitly by the transport Equation (2) in each time step-the velocity $\mathbf{u}$ is obtained from $p$ according to Equation (3). Using an FE discretization for $p$ (linear basis functions) and $\mathbf{u}$ (constant basis functions), a Jacobi-preconditioned CG solver [Shewchuk 1994] computes the new pressure distribution until the preconditioned residual norm is smaller than $10^{-5}$ (using $p$ from the previous time step as the starting condition, typically around 20 iterations are sufficient). Stricter bounds did not improve the result in our test cases. Alternatively, $\operatorname{sam}(\mathrm{oa})^{2}$ also allows one to choose a relative criterion based on the start residual. For the first time step, the initial guess for $p$ is obtained following a nested iteration approach; that is, to limit the number of iterations, we successively solve for $p$ and interpolate to a refined grid during grid setup.

For the transport equation, a dual-grid (or node-centered) FV method was used, where $S$ is stored on the grid vertices, which act as dual cell centers. The numerical flux is computed with the upwind method. Because $F \geq 0$ this is valid, even though the flux is nonlinear. Here, the main advantage of the dual grid approach becomes apparent, as $\mathbf{u}$ is well defined on the dual-cell intersections. Since the velocity is constant in all primal cells, it is piecewise constant in the dual cell intersections, which are perpendicular to the primal cell intersections. Note that the dual grid is not generated explicitly, but consists of patches of intersecting primal cells instead. It is thus implemented using only volume and DoF operators, as provided by $\operatorname{sam}(\mathrm{oa})^{2}$.

Fused CG Solver for the Pressure Equation. Algorithm 2 (left) sketches the kerneloriented implementation of the CG solver (with diagonal preconditioning) in $\operatorname{sam}(\mathrm{oa})^{2}$. Due to the data dependency between the dot products and updates, two grid traversals are required for each iteration. For a memory-bound solver, this is unsatisfactory as in both traversals all data is touched, but only half of the unknowns are updated. Better performance could be achieved if all operations were performed in a single traversal.

We therefore adopted a CG variant by Saad [1985] and Meurant [1987] that allows overlapping of the matrix-vector product $u \leftarrow A d$ and the application of the diagonal preconditioner $v \leftarrow D^{-1} u$. This version requires only a single traversal per iteration without extra helper vectors. Its implementation in $\operatorname{sam}(\mathrm{oa})^{2}$ is given in Algorithm 2 (right). Its main idea is to precompute $\beta=\frac{\gamma}{\gamma_{\text {old }}}$ without knowledge of the new residual by using

$$
\gamma=r^{T} D r-2 \alpha v^{T} D r+\alpha^{2} v^{T} D v .
$$

The search vector and solution update can be overlapped now, as both $\alpha$ and $\beta$ are known. Two extra dot products appear at this point, one of which is redundant due to $D$-orthogonality of the preconditioned residuals. With this, the equation reduces to

$$
\gamma=\alpha^{2} v^{T} D v-r^{T} D r .
$$

Hence, only one extra dot product is required per iteration compared to classical CG. Note that the indirect update of $\gamma$ technically reduces the accuracy of the algorithmwe did not observe an influence on convergence in our tests, however. At convergence, fused CG requires one extra iteration that is caused by postponing the solution update to the following iteration. Still, the total number of traversals is roughly halved, which outweighs the disadvantage of an additional dot product.

Mass Conservative Refinement. While interpolation of the pressure $p$ to a refined or coarsened grid corresponds to a change of basis of the FE solution, using a similarly 
ALGORITHM 2: Comparison of Classical CG and Fused CG with Diagonal Preconditioning: Classical CG Requires Two Grid Traversals and Three Dot Products Per Iteration; Fused CG Executes Only One Grid Traversal Per Iteration but Computes an Extra Dot Product.

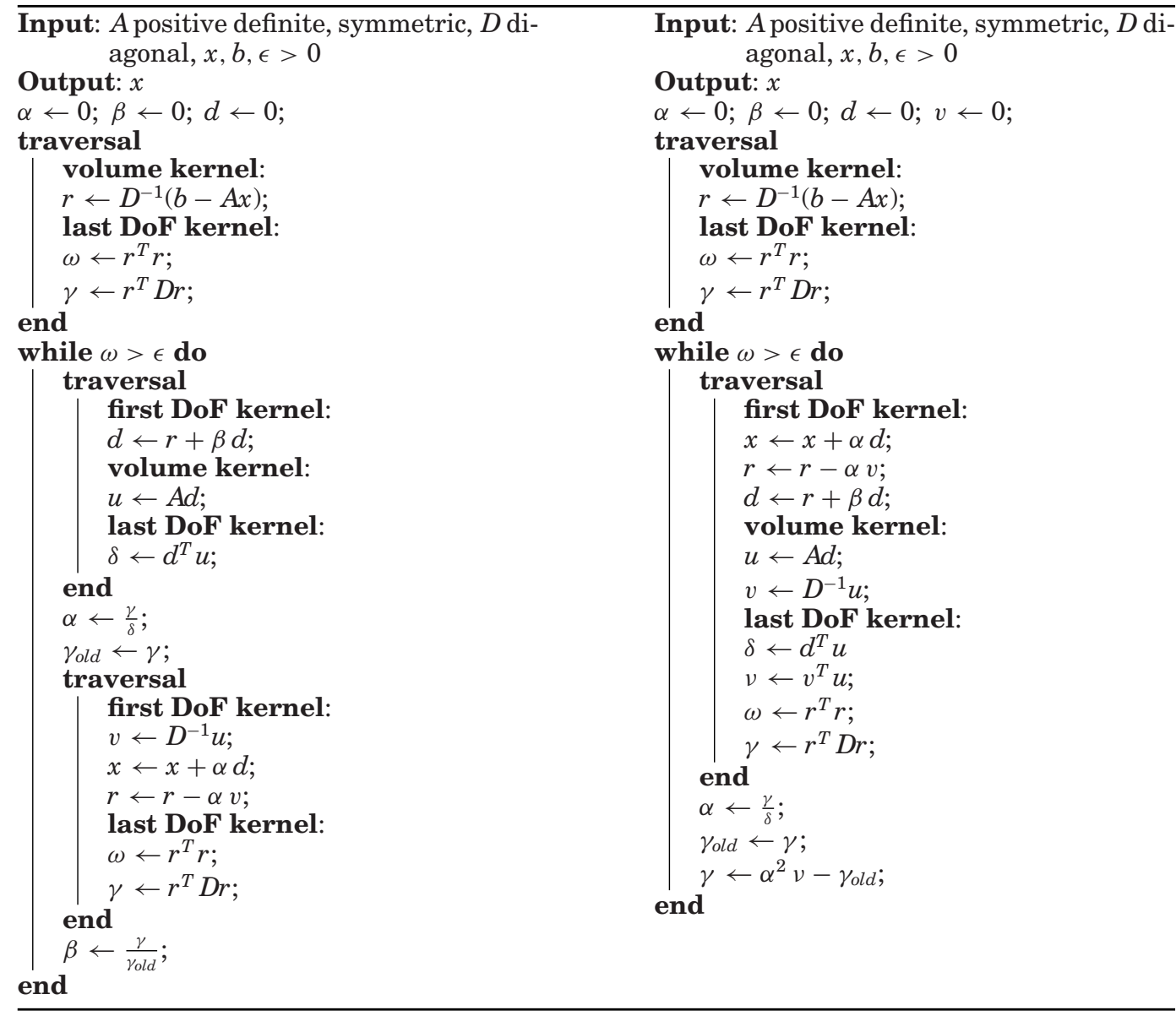

straightforward interpolation for $S$ does not guarantee conservation of mass, such that global quantities of interest (e.g., accumulated oil production) would likely be wrong. While the mass $m_{\alpha}=\rho_{\alpha} \Phi S_{\alpha}$ of a phase $\alpha$ cannot be conserved pointwise, FE theory suggests that weak conservation between the old and new grid can be achieved. Assume shape functions $\hat{\phi}$, porosity $\hat{\Phi}$, and saturation $\hat{S}$ associated with the old grid, and similarly $\phi, S, \Phi$, and a test basis $\psi$ associated with the new grid. Weak formulation and Galerkin projection for both phases then results in two equivalent LSEs:

$$
\begin{aligned}
& \rho_{w} \mathbf{M}_{\phi, \psi} \Phi_{\phi} \mathbf{S}_{\phi}=\rho_{w} \mathbf{M}_{\hat{\phi}, \psi} \hat{\Phi}_{\hat{\phi}} \hat{\mathbf{S}}_{\hat{\phi}} \\
& \rho_{n} \mathbf{M}_{\phi, \psi} \Phi_{\phi}\left(\mathbf{1}-\mathbf{S}_{\phi}\right)=\rho_{n} \mathbf{M}_{\hat{\phi}, \psi} \hat{\Phi}_{\hat{\phi}}\left(\mathbf{1}-\hat{\mathbf{S}}_{\hat{\phi}}\right) .
\end{aligned}
$$

The mass matrix in the Left Hand Side (LHS) is diagonal due to the chosen FV bases $\phi, \hat{\phi}$, and $\psi$. Effectively, we therefore need to compute the saturation of each FV cell on the new grid by averaging all saturations of FV cells on the old grid, weighted by the intersecting volumes of water in the old and new cell. Algorithm 3 shows how this is implemented via the specific $\operatorname{sam}(\mathrm{oa})^{2}$ kernels. All kernels perform the same operation, 

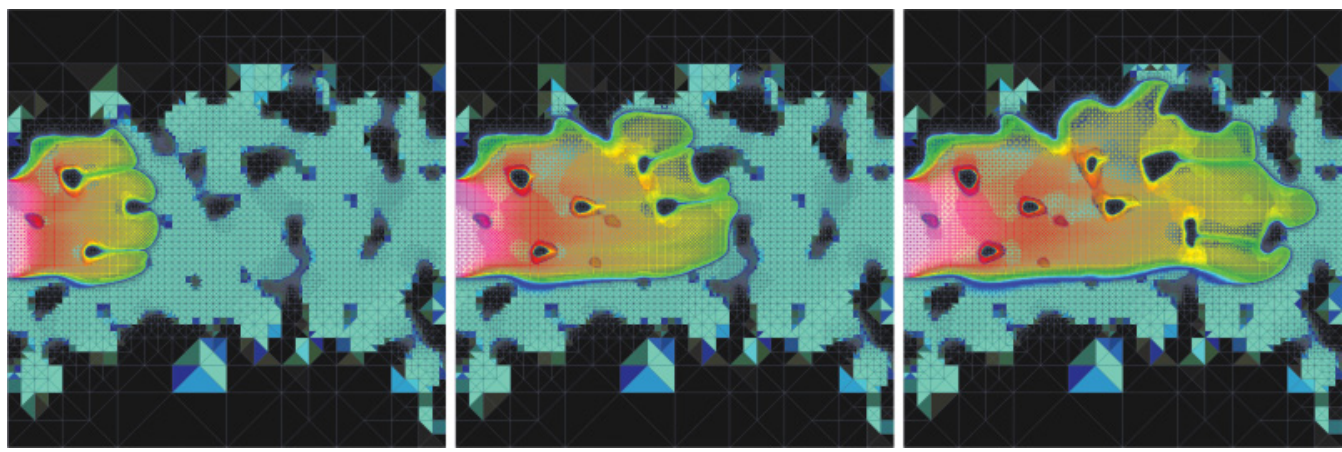

Fig. 7. Porous media flow: Water enters a heterogeneous porous medium from the left, replacing an oil phase as time progresses. Dark areas are impermeable; light blue areas are permeable. The grid is refined at the saturation front for a sharply defined shock wave and coarsened in all other areas.

ALGORITHM 3: Mass Conservative Saturation Transfer from Old Grid (Marked by Cell Volume $\hat{V}_{j}$, Porosity $\hat{\Phi}_{j}$, and Saturation $\left.\hat{S}_{j}\right)$ to New Grid $\left(V_{i}, \Phi_{i}, S_{i}\right)$. Saturation $S_{j}$ in Cell $j$ Is the Average Over the Saturations $\hat{S}_{j}$ of All Old Cells $i$ That Intersect with the New Cell, Weighted by the Intersecting Volumes of Water.

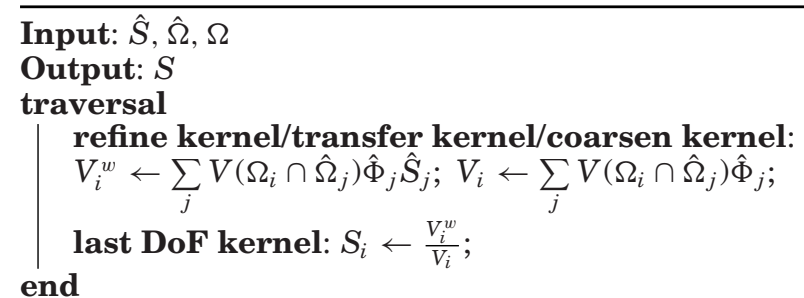

but while the source and destination element coincide in the transfer kernel, they do not in refinement and coarsening kernels, resulting in different intersection volumes.

Test Scenario. As a benchmark setting for our porous media flow mode, we separated the computational domain into a large free-flow area with chunks of impermeable material arranged in a pseudo-random pattern and smooth material transitions in between. Figure 7 shows such a channel flow scenario with heterogeneous permeabilities similar to the test cases of Chueh et al. [2010]. Typical behavior of nonlinear fluxes is observed as a shock is built at the wavefront from saturation 0 to 0.3 , followed by a rarefaction wave from saturation 0 . to 1 . Adaptive refinement is used to keep the interface sharp at the shock front. The rarefaction wave behind is coarsened again. The local change of saturation was used as an error indicator for refinement. Coarsening was triggered if the saturation was nearly constant in two adjacent elements. The library ASAGI (A Parallel Server for Adaptive Geoinformation [Rettenberger 2012], https://github.com/TUM-I5/ASAGI) was used as a parallel server to provide the material data required for mesh refinement.

Discretization on a fine grid and further a priori refinement of the transitions generate an initial mesh of roughly 70 million cells.

Limitations. We selected the presented scenario to showcase the efficiency of parallel adaptive mesh refinement for porous media flow and to examine the performance of models with a dominant memory-bound component (the CG solver). A multitude of model extensions would be required for production-ready simulations, such as 
anisotropic permeabilities, 3D domains, miscible flow, multiphase flow, gravity, and so forth. We consider these extensions to be beyond the scope of this article. In recently published work [Meister and Bader 2015], we implemented an enhanced model to solve the SPE10 benchmark on a 3D prism grid using $2 \mathrm{D}$ adaptivity in $\operatorname{sam}(\mathrm{oa})^{2}$.

For production scenarios, permeability $K$ can be anisotropic and strongly heterogeneous, leading to highly ill-conditioned pressure (Equations (4)), requiring sophisticated numerical solvers and stopping criteria. In our matrix-free setup, geometric multigrid solvers would be the method of choice. While multigrid methods can be implemented via the Sierpinski traversal approach [Bader et al. 2008], robust multigrid methods for Poisson problems with strongly heterogeneous coefficients are a challenge of their own and therefore not considered in this article.

\subsection{Tsunami Wave Propagation}

Our second test case is a tsunami wave propagation scenario, using a simplified model based on the 2D shallow water equations as described in, for example, George [2008]:

$$
\begin{gathered}
h_{t}+(h u)_{x}+(h v)_{y}=0 \\
(h u)_{t}+\left(h u^{2}+\frac{1}{2} g h^{2}\right)_{x}+(h u v)_{y}=-g h b_{x} \\
(h v)_{t}+(h u v)_{x}+\left(h v^{2}+\frac{1}{2} g h^{2}\right)_{y}=-g h b_{y} .
\end{gathered}
$$

This hyperbolic system of PDEs describes the conservation of height $h$ (equivalent to mass) and momentum $\mathbf{p}=(h u, h v)^{T}$ of an inviscid flow in a shallow domain with bathymetry $b$. "Shallow" in this context means that the domain (especially the characteristic wave lengths) in $\mathrm{x}$ - and $\mathrm{y}$-dimensions is large compared to the extent in the $\mathrm{z}$-dimension (ocean depth). The equations may be derived from the 3D Euler equations by integration over the z-axis, such that depth-averaged values for velocity and momentum are used instead of a full 3D model.

Finite Volume Discretization. For a numerical solution, we adopt an FV approach by LeVeque et al. [2011]. Unknowns are represented via cell-averaged values in each element. The respective element-local vector $Q_{i}^{n}$ contains the cell-averaged water height and momentum in element $i$ at time $n$. Following a wave propagation approach, socalled net updates $\mathcal{F}^{\text {in }}\left(Q_{i}^{n}, Q_{j}^{n}\right)$ are computed on the cell-incident edges. The net updates represent transport of mass and of momentum into and out of the elements via a given edge, which leads to an update scheme similar to an explicit Euler time step:

$$
Q_{i}^{n+1}=Q_{i}^{n}+\frac{\Delta t}{\Delta x} \sum_{j \in \mathcal{N}(i)} \mathcal{F}^{\text {in }}\left(Q_{i}^{n}, Q_{j}^{n}\right) .
$$

The equation puts the change of a quantity vector $Q_{i}^{n}$ in relation to bidirectional fluxes that act on the element boundaries. A time step thus consists of evaluating fluxes on all element boundaries and accumulating a net update in each element. To compute the net updates $\mathcal{F}^{\text {in }}\left(Q_{i}^{n}, Q_{j}^{n}\right)$, the corresponding Riemann problem needs to be solved on all element boundaries, where $Q_{i}^{n}$ and $Q_{j}^{n}$ are the quantities of cells incident to the respective edges. $\operatorname{sam}(\text { oa })^{2}$ uses the approximate Riemann solvers provided by the package GeoClaw [LeVeque 2002], which are integrated on the kernel layer. For our simulation scenario, we applied an augmented Riemann solver [George 2008], which is tailored to the concrete requirements of tsunami simulation. In particular, it is able to correctly handle wetting and drying to capture inundation of coasts. 

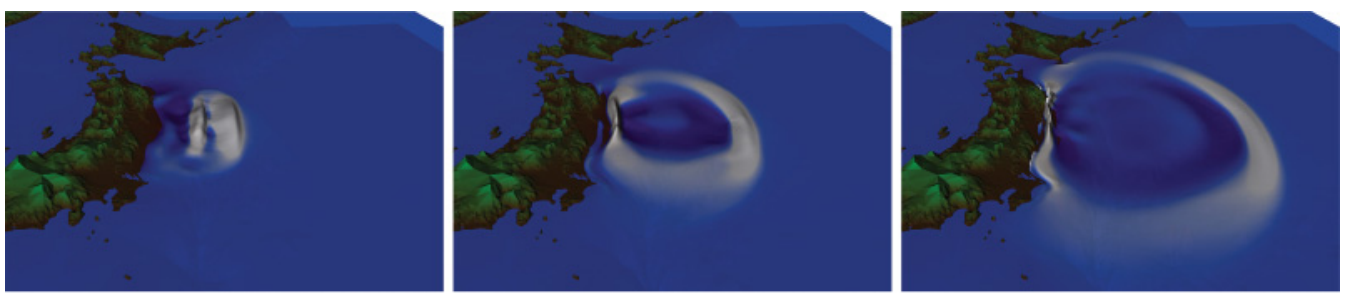

Fig. 8. Tsunami wave propagation: An earthquake in Tohoku, Japan, in 2011 causes a vertical fault displacement and creates a tsunami wave that propagates radially from the fault. The wave is displayed at 6 minutes (left), 30 minutes (middle), and 54 minutes (right) after the earthquake.

ALGORITHM 4: Time-Stepping Scheme for the Tsunami Wave Propagation Scenario. For Each Cell-Edge Pair, the Quantity Vector Is Copied to the Edge, Then the Net Updates Are Computed on Each Edge, and Finally the Quantity Vector Is Updated with the Accumulated Net Updates.

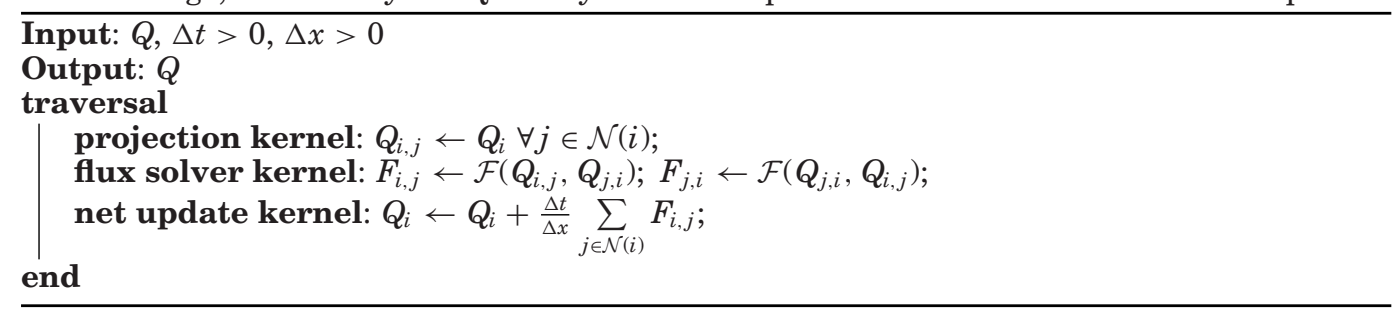

In $\operatorname{sam}(\mathrm{oa})^{2}$, the resulting time-stepping scheme is performed as described in Algorithm 4 by implementation of a Cell-to-Edge Operator, which computes a representation of the state vector on the incident edges. For piecewise constant data, this corresponds to copying or creating a reference to the cell data. The Skeleton Operator calls a flux function on each edge, which solves the 1D Riemann problem on the edge to compute net updates. Finally, the Cell Update operator accumulates the net updates from all edges of a cell to compute an update for the cell unknowns.

Test Scenario. To obtain a realistic simulation scenario, we used bathymetry data of the Northern Pacific and the Sea of Japan (The GEBCO_08 Grid, version 20100927) in order to simulate the Tohoku tsunami of 2011. The data is projected by a lengthpreserving mapping, thus taking into account the curvature of the earth. As source terms, we used time-dependent displacements obtained from a simulation of the Tohoku earthquake by Galvez et al. [2013]. The subsequent tsunami propagation as well as inundation of the Japanese coast is simulated by sam $(\text { oa })^{2}$ (see Figure 8). Dynamical refinement along wavefronts and coarsening in wake regions are triggered via an error indicator based on momentum change in each element. Again, bathymetry and displacement data were handled by ASAGI.

Interpolation of unknowns during grid refinement is not straightforward. As described in LeVeque et al. [2011], for example, interpolation of water height $h$ and bathymetry $b$ needs to be carefully chosen to avoid spurious waves, even at the cost of sacrificing exact conservation of mass of momentum. Therefore, interpolation is oriented toward a correct interpolation of the water surface $b+h$ and then inferring $h$ from the new bathymetry data.

During the simulation, the mesh size reaches 1 million elements for depth 24 and 16 million elements for depth 28 . These numbers change strongly during the simulation due to frequent remeshing. The wall-clock times for this scenario were approximately 3 and 30 minutes, respectively, on 512 SuperMUC cores. 


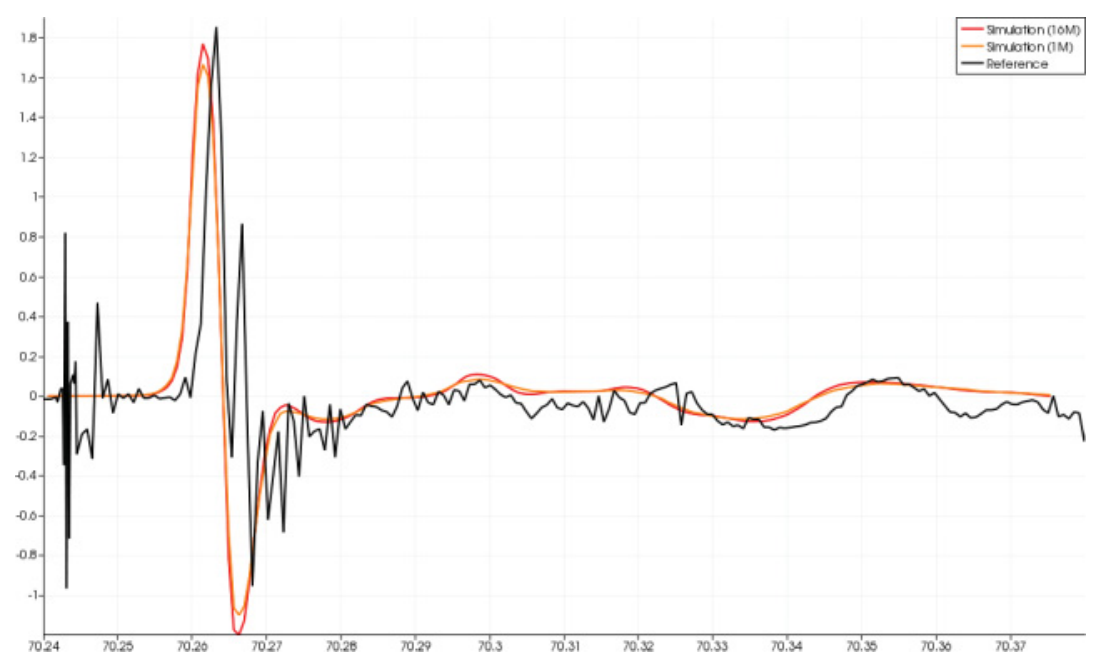

Fig. 9. Measured (black) and simulated (orange: max. depth 24, red: max. depth 28) water displacement over time at the location of DART buoy 21418 (http://www.ngdc.noaa.gov/hazard/dart/2011honshu_dart.html).

We compared our results with respective water displacements measured by DART buoys (see Figure 9). The observed phase displacement and amplitude differences are due to model and input data restrictions; high-frequency oscillations in the reference data are mostly from surface waves and noise.

Limitations. In our shallow water model, we neglected additional source terms, such as the Coriolis Force or bottom friction, as their effect on adaptive mesh refinement as well as their impact on parallel performance is small. Current work includes the implementation of nonhydrostatic models, which requires the solution of a global LSE for nonhydrostatic pressure in each time step (similar to the porous media flow scenario). Respective model extensions are known to affect the solution, especially in coastal regions [Horrillo et al. 2006]. Note that sam(oa $)^{2}$ does not efficiently support reconstruction of flux functions with large stencils, as they are required by many high-order FV methods. Hence, high-order discretization in sam(oa ${ }^{2}$ should adopt DG approaches.

\section{PERFORMANCE RESULTS}

We analyzed the performance of $\operatorname{sam}(\mathrm{oa})^{2}$ on SuperMUC, a Petascale system with more than 147k Intel Sandy Bridge cores and a peak performance of 3 PFLOPS (https://www.lrz.de/services/compute/supermuc/systemdescription/). It uses an islandoriented fat-tree topology with intraisland nonblocking Infiniband communication and a 4:1 pruned interisland connection. Scalability for distributed memory was tested on the SuperMUC "thin nodes," each of which contains two Intel Xeon E5-2680 processors (eight cores, 2.4GHz, 20MB shared L3, 32GB shared memory). For shared-memory scalability tests, we used the SuperMUC "fat nodes," which consist of Intel Xeon E74870 processors (four sockets, each with 10 cores, $2.4 \mathrm{GHz}, 24 \mathrm{MB}$ shared L3, 256GB shared memory). Unless mentioned otherwise, we used the Intel Fortran Compiler 13.1 and Intel MPI 4.1 provided on SuperMUC, and used double-precision arithmetics. All our tests, especially speedup tests, aim at providing a simulation environment similar to a production run and hence include adaptive mesh refinement and load balancing after each time step.

Efficiency Metrics. For simulations on dynamically adaptive meshes, the classical weak-scaling requirement of having a constant number of unknowns per processor 

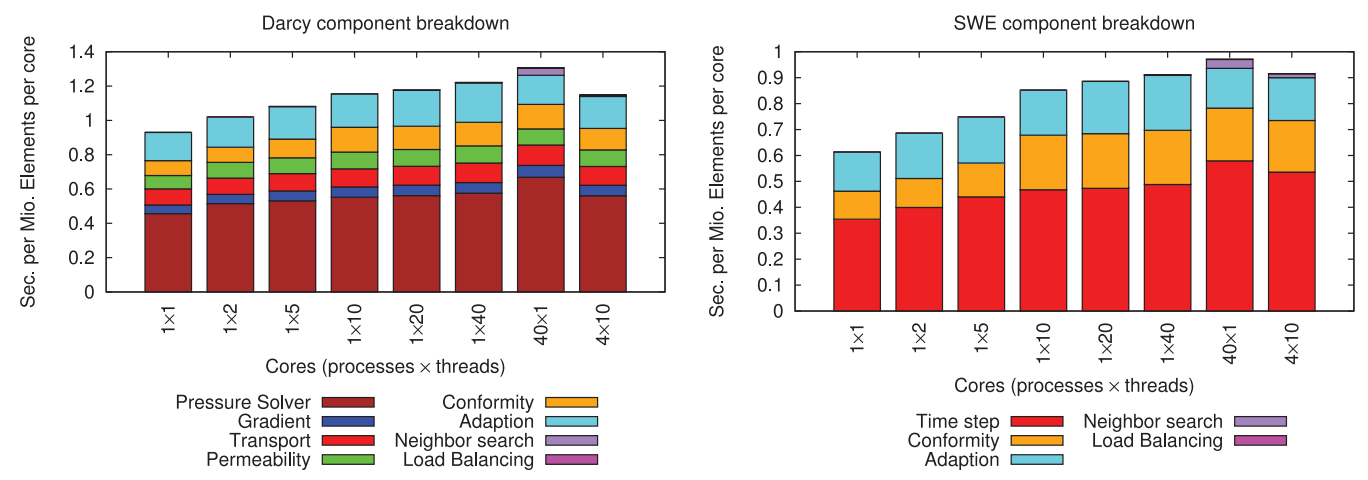

Fig. 10. Hybrid strong scaling with 40M unknowns on a single SuperMUC fat node. A component breakdown is plotted for the porous media flow scenario (left) and the tsunami scenario (right) for pure OpenMP (one to 40 cores), pure MPI (40 ranks $\times 1$ core), and hybrid parallelization ( 4 ranks $\times 10$ cores). Pure OpenMP suffers from NUMA effects caused by the underlying ASAGI library, which affects the performance of adaptive refinement in both scenarios. Pure MPI suffers from imbalance, caused by nonuniform kernel execution times. Hybrid parallelization (with one MPI rank per socket) performs best in both cases, as load balance is improved and NUMA effects do not occur.

(core, node, ...) is difficult to achieve. We therefore used the number of element touches per second per core, $\mu(p)=\frac{E T(p)}{p \cdot t(p)}$, as the parallel performance measure for $\operatorname{sam}(\mathrm{oa})^{2} . t(p)$ is the wall-clock time measured on $p$ cores, and $E T(p)=\mu(p) t(p) p$ is the total number of element touches during the simulation-each grid traversal "touches" all elements once. For a strong scaling setting, $E T(p)=E T(1)=\mu(1) t(1)$ is independent of $p$ and $\mu(p)=\frac{t(1)}{p \cdot t(p)} \mu(1)$ becomes equivalent to parallel efficiency scaled by the constant $\mu(1)$. In a weak scaling setting, parallel efficiency is usually measured as $\frac{t(1)}{t(p)}$ with the condition that the problem size per core is constant. However, dynamically adaptive grids cannot easily meet this condition. In contrast, $\mu(p)$ is independent of the problem size and thus provides a better metric.

For component breakdowns, we measured $\tau(c, p)=\frac{p \cdot t(c, p)}{E T(p)}$, where $t(c, p)$ is the total wall-clock time spent in each component $c$ and $E T(c, p)$ is the number of element touches per component. Hence, $\tau(c, p)$ measures the average wall clock time per element-including structural and parallel overhead-for each solver component $c$ per simulation time step.

\subsection{Scalability and Performance on a Shared-Memory Compute Node}

$\operatorname{sam}(\mathrm{oa})^{2}$ provides a set of different approaches for concurrent execution, based on pure OpenMP, pure MPI, and hybrid parallelization. We performed a direct comparison on a shared memory system in order to directly compare all three variants. Additionally, results on single-node execution were used to determine a performance baseline for parallel efficiency on distributed systems.

5.1.1. Comparison of OpenMP, MPI, and Hybrid Parallelization. A SuperMUC fat node consists of four NUMA sockets with 10 cores each, suitable for strong scaling tests with pure OpenMP, pure MPI, and hybrid OpenMP/MPI parallelization. The benefit of this system is that a direct comparison of all approaches is possible for up to 40 cores.

Strong Scaling. Figure 10 shows a component breakdown to evaluate the parallel efficiency for both scenarios from one to 40 cores. In each time step of the porous media flow scenario, the linear system is set up first and relative permeabilities are computed (Permeability). Then the system is solved (Pressure Solver) and the pressure 
Table I. OpenMP Scheduling Strategies

\begin{tabular}{lccc}
\hline Scenario & Sections & Work Sharing & Work Stealing \\
\hline Porous Media Flow & 16 & $33.02 \mathrm{~s}$ & $32.66 \mathrm{~s}$ \\
& 32 & $35.06 \mathrm{~s}$ & $34.55 \mathrm{~s}$ \\
Tsunami & 16 & $78.19 \mathrm{~s}$ & $71.37 \mathrm{~s}$ \\
& 32 & $82.28 \mathrm{~s}$ & $74.24 \mathrm{~s}$ \\
\hline
\end{tabular}

Wall-clock times for eight MPI processes with 10 threads each: The porous media flow scenario is well balanced with OpenMP work sharing due to its homogeneous kernel calls and does not gain performance by work stealing. The tsunami scenario suffers from branched kernels. Work stealing therefore provides a significant performance increase.

gradients are used to compute the time step size (Gradient). Next, the transport step (Transport) is executed and new refinement and coarsening flags are set. The flags are iteratively corrected to restore a conforming grid (Conformity) and then remeshing is done (Adaption). This process includes regeneration of communication structures (Neighbor Search). Finally, the grid must be rebalanced among threads and processes (Load Balancing).

Performance of the porous media scenario is mostly affected by the Poisson solver for the pressure equation in each time step. In Figure 10, we normalized the respective component to 10 solver iterations, as the actual number may differ between problem sizes. Relative permeability computation, pressure gradient computation, advective transport, and adaptive refinement make up the remaining numerical traversals. Additionally, the plot shows how core components, such as conformity traversals, load balancing, and reconstruction of MPI communication structures, affect performance.

The tsunami scenario executes the transport step (Time Step) in each time step first. It includes flux computation, state updates, and setting refinement and coarsening flags. As in the porous media scenario, the following steps are conformity correction (Conformity), remeshing (Adaption), regeneration of communication structures (Neighbor Search), and load balancing (Load Balancing).

Pure OpenMP parallelization suffers slightly from NUMA effects caused by the underlying ASAGI library, which is visible in the initial displacement for the tsunami scenario and adaptive refinement for both scenarios. Pure MPI parallelization is imbalanced due to inhomogeneous kernel execution times, which are more prominent in the tsunami scenario. Hybrid parallelization solves both issues well enough to beat pure OpenMP and pure MPI in both scenarios.

OpenMP: Work Stealing Is Better Than Work Sharing. sam(oa $)^{2}$ implements static and dynamic task-based scheduling of OpenMP threads. The latter allows threads to steal work from other threads once their tasks are finished. Due to the inhomogeneous flux solver kernel in the tsunami scenario, this scenario benefits strongly from taskbased scheduling as shown in Table I. However, even the inherently balanced porous media flow kernels profit to a small degree from task stealing. Inhomogeneity is caused by imperfect load balancing and latency from reading external data in this case. As work stealing performs better, we used it for all following tests.

5.1.2. Baseline Efficiency. In order to define a baseline for parallel efficiency, we executed both application scenarios on a single SuperMUC thin node, which consists of 16 cores. Memory throughput was measured by counting the total amount of data accessed in the traversal and dividing by the traversal time. Only one read access to each data was counted. We thus reflect that $\operatorname{sam}(\text { oa })^{2}$ accesses the same stream for read and write operations (not necessarily at the same location though) and consider all stack accesses to be cached. Hence, all given performance measurements are conservative 
Table II. Single-Node Memory Performance

\begin{tabular}{lcccc}
\hline Scenario & Mem TP & Ratio & L1 Hits & CPMI \\
\hline sam $(\text { oa })^{2}$ & $20.2 \mathrm{~GB} / \mathrm{s}$ & $48 \%$ & $99.7 \%$ & 1.33 \\
STREAM & $42.6 \mathrm{~GB} / \mathrm{s}$ & $100 \%$ & $64.2 \%$ & 2.72 \\
\hline
\end{tabular}

Single-node performance of a Poisson stencil computation compared to an in-place vector scaling performed by the STREAM benchmark. Both $\operatorname{sam}(\mathrm{oa})^{2}$ and STREAM count only one access per data per iteration for this comparison.

estimates that count only compulsory accesses to main memory. We compared the respective memory throughput with results from the STREAM [McCalpin 1995] benchmark. Floating-point performance is evaluated via application-specific measures.

Porous Media Flow. The performance of the porous media flow scenario is dominated by the linear pressure solver, which has a small arithmetic intensity. We thus expect a memory-bound situation and aim for high memory throughput. Table II gives an overview of performance results determined by a parallel run on a single node (simulation with $5 \mathrm{M}$ cells and $280 \mathrm{MB}$ data). Memory throughput is measured by dividing the amount of allocated memory by the wall clock time of a single Poisson iteration. The number in parentheses is the ratio to STREAM performance.

For single-node performance, $20 \mathrm{~GB} / \mathrm{s}$ memory throughput or $48 \%$ compared to the STREAM benchmark is achieved. This measurement is conservative, since STREAM accesses each array element only once, whereas $\operatorname{sam}(\mathrm{oa})^{2}$ may touch elements multiple times. The performance of the scenario is thus limited to a factor of 2 on a single node as the hardware permits a higher memory throughput.

The table also includes the ratio of Level 1 cache hits and the average number of Cycles Per Memory Instruction (CPMI). Ideally the cache hit rate is 100\%, meaning all data access happens from cache. The CPMI count measures the mean number of cycles required per memory access. If memory access is exclusively from registers, this number will be 1; otherwise, it will be higher. Both numbers are close to optimal. Most data is accessed multiple times, but since it is usually located in registers or caches, memory calls have little latency. The STREAM benchmark accesses each data only once and thus returns a higher throughput, a lower cache hit rate, and more CPMI.

Another comparative metric for this scenario is the element throughput that describes the speed at which $\operatorname{sam}(\mathrm{oa})^{2}$ can process elements that execute numerical operators. For the Fused CG solver, the result is $24 \mathrm{M} / \mathrm{s}$ in serial and $360 \mathrm{M} / \mathrm{s}$ in parallel execution (on 16 cores). If all other components of the solver are included, we obtain $20 \mathrm{M} / \mathrm{s}$ in serial and $286 \mathrm{M} / \mathrm{s}$ in parallel execution. All element touches that contain numerical operations are counted, including adaptive refinement, as it performs interpolation of unknowns. Computation is dominated by the Poisson solver with 22 iterations per time step and $65 \%$ of the execution time, whereas all other components each perform one or two iterations per time step and add up to the remaining $35 \%$.

Tsunami Wave Propagation. The performance of the tsunami wave propagation scenario is dominated by the Riemann solver called for all edges. A detailed performance study of the augmented Riemann solver used in our simulations was executed in Bader et al. [2014]. The respective study was based on the Cartesian mesh code SWE (https://github.com/TUM-I5/SWE/ (SWE)) and used a slightly simplified, singleprecision $\mathrm{C} / \mathrm{C}++$ implementation of the augmented Riemann solver, in contrast to the GeoClaw solver used by sam $(\mathrm{oa})^{2}$. Both Riemann solvers cannot be vectorized by the compiler. The SWE solver was shown to be computation bound. We thus use the SWE performance (on a regular mesh) as an estimate of the achievable performance for our dynamically adaptive tsunami simulation scenario. 
Table III. Local vs. Global Load Propagation

\begin{tabular}{lccccc}
\hline & & \multicolumn{2}{c}{ Local Load Propagation } & \multicolumn{2}{c}{ Global Load Propagation } \\
Scenario & MPI ranks & Time (ratio) & Total Time & Time (ratio) & Total Time \\
\hline PMF & 64 & $0.12 \mathrm{~s}(0.4 \%)$ & $29.40 \mathrm{~s}$ & $0.06 \mathrm{~s}(0.2 \%)$ & $29.63 \mathrm{~s}$ \\
& 128 & $0.09 \mathrm{~s}(0.6 \%)$ & $15.23 \mathrm{~s}$ & $0.04 \mathrm{~s}(0.2 \%)$ & $15.35 \mathrm{~s}$ \\
& 256 & $0.08 \mathrm{~s}(1.0 \%)$ & $7.99 \mathrm{~s}$ & $0.02 \mathrm{~s}(0.3 \%)$ & $8.00 \mathrm{~s}$ \\
Tsunami & 64 & $3.56 \mathrm{~s}(2.6 \%)$ & $136.60 \mathrm{~s}$ & $1.23 \mathrm{~s}(0.9 \%)$ & $134.64 \mathrm{~s}$ \\
& 128 & $3.81 \mathrm{~s}(5.3 \%)$ & $71.59 \mathrm{~s}$ & $0.98 \mathrm{~s}(1.4 \%)$ & $69.15 \mathrm{~s}$ \\
& 256 & $5.25 \mathrm{~s}(13 \%)$ & $40.54 \mathrm{~s}$ & $0.87 \mathrm{~s}(2.4 \%)$ & $36.45 \mathrm{~s}$ \\
\hline
\end{tabular}

For test cases with few time steps, the global variant scales better and is faster by a factor of 3 to 6 on 256 cores.

In order to obtain comparable results, we compiled sam $(\text { oa })^{2}$ with single precision and counted the total number of Riemann solves divided by the wall-clock time for both codes. The total time in $\operatorname{sam}(\mathrm{oa})^{2}$ therefore includes overhead for adaptivity. Our measurements show that $\operatorname{sam}(\mathrm{oa})^{2}$ performs $3.2 \mathrm{M}$ Riemann solutions per second in serial execution. We executed a serial version of https://github.com/TUM-I5/SWE/ (SWE) on the same system and a similar problem and measured 6.8M Riemann solutions per second. Thus, computation on an adaptive grid in $\operatorname{sam}(\mathrm{oa})^{2}$ is around half as fast as on a Cartesian grid in SWE. Figure 10 shows that around 55\% to $60 \%$ execution time in $\operatorname{sam}(\mathrm{oa})^{2}$ is spent on the time step, while $35 \%$ to $40 \%$ is required for adaptive refinement and coarsening. Hence, a significant ratio of time is required for dynamical adaptivity, causing most of the performance loss compared to SWE.

Parallel execution of $\operatorname{sam}(\mathrm{oa})^{2}$ results in $43 \mathrm{M}$ Riemann solutions per second.

\subsection{Scalability on a Distributed-Memory Supercomputer}

For multinode performance tests, the SuperMUC thin nodes were used. Each node contains only 16 cores, which causes memory banks to be more distributed and parallel performance to depend mostly on MPI parallelization. Since problem sizes of 2D scenarios are usually limited to $10 \mathrm{M}$ to $100 \mathrm{M}$ DoFs, the goal is to get good performance up to possibly 500 to 1,000 cores for these scenarios. We therefore compared MPI load-balancing strategies and conducted scaling tests on up to 8,192 cores in order to examine parallel efficiency on a multinode system.

5.2.1. Global and Local Load Propagation. Two load propagation strategies were implemented in $\operatorname{sam}(\mathrm{oa})^{2}$ : a local approach that allows migration only between processes with successive ranks, and a global strategy that allows arbitrary migration between any processes. The local approach causes little global communication but handles strong changes inefficiently due to detoured communication, while the global approach has less communication volume but may cause more latency. In order to demonstrate behavior of both strategies on scenarios with a dominant initial phase, we compared load-balancing times to total times (wall clock) for the porous media flow scenario with 10 semi-implicit time steps and the tsunami scenario with 100 explicit time steps respectively on 64,128 , and 256 MPI ranks (Table III). It is apparent that global load balancing performs much better, as performance is improved by a factor of up to 6 . For longer execution times, the speedup is smaller but still significant. We therefore chose the global variant for all our tests.

5.2.2. Strong Scaling. To test the parallel speedups achieved for a given scenario with a fixed size, we picked a porous media flow and a tsunami scenario with $60 \mathrm{M}$ to $100 \mathrm{M}$ elements and studied performance on 16 to 2,048 cores (Figure 11). Also displayed are results of hybrid parallelization with one MPI process per socket and eight OpenMP threads per process. No significant difference is observed, however. 

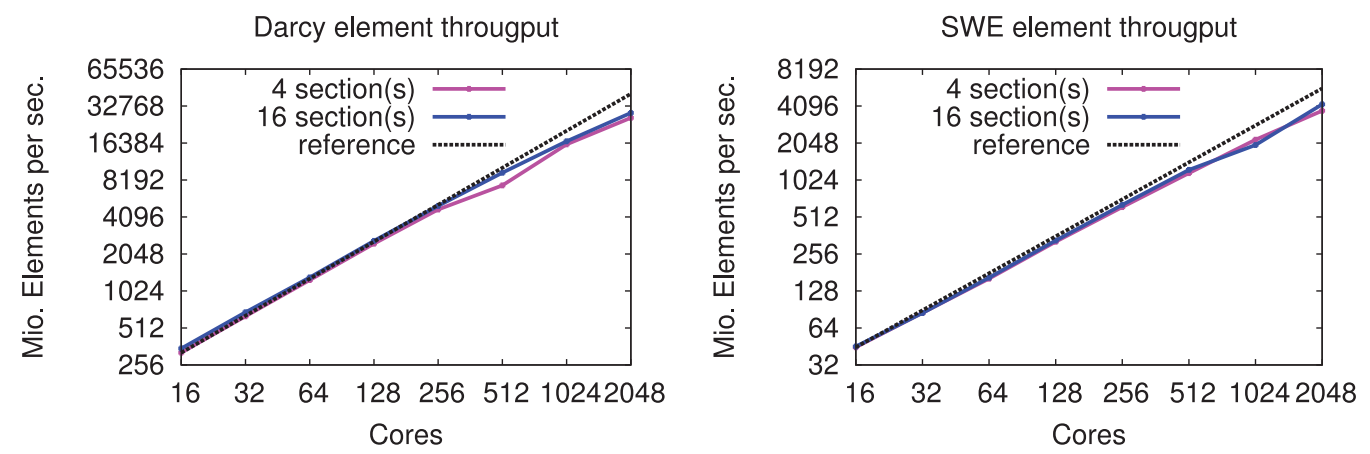

Fig. 11. Pure MPI (blue, 16 sections per core) and Hybrid OpenMP/MPI (pink, four sections per core) strong scaling with $60 \mathrm{M}$ to $100 \mathrm{M}$ unknowns on the SuperMUC thin nodes for porous media flow (left) and tsunami wave propagation (right). Hybrid parallelization scales worse for porous media flow and slightly better for tsunami simulation. The porous media flow scenario used 14 refinement depth levels with a maximum depth of 40; tsunami wave propagation used refinement depths of 24 refinement depth levels with a maximum depth of 32 .

The porous media scenario scales from 16 to 512 cores with a parallel efficiency of $88 \%$; the tsunami scenario reaches $86 \%$. Performance loss is mostly due to load imbalance. Homogeneity is assumed in the load estimate; however, for the tsunami scenario, the flux solver kernel has an early-exit condition and thus smaller execution times for water-free regions. Other load-balancing techniques could be applied here for better load estimates. We have found that switching to timing-based load balancing downgrades performance though, because structural components like conformity checks and grid refinement rely on evenly distributed elements in parallel.

For 1,024 to 2,048 cores, global communication in the CG solver starts to affect performance. Parallel efficiency of the porous media flow scenario drops to $65 \%$. At 2,048 cores, traversal time per core is already reduced to less than $3 \mathrm{~ms}$. Here the problem size per core (30k elements per core) becomes much too small for a memorybound problem (see, e.g., Weinzierl et al. [2013]). As the tsunami scenario has longer traversal times with $30 \mathrm{~ms}$ at 2,048 cores, it is not as heavily affected and parallel performance is reduced only to $73 \%$.

5.2.3. Weak Scaling. Our final test is a weak scaling study performed with the porous media flow and the tsunami scenario using up to 8,192 cores (one SuperMUC thin node island) and $10 \mathrm{G}$ elements, in order to predict performance of larger problems that may occur in future simulations. For our measurements, we skipped grid setup and the first time steps in order to simulate sustained performance of a production run. Initially the grid is incrementally refined and distributed until it is fully partitioned and fully resolved up to an error indicator. This initial phase is thus inherently imbalanced and causes increased data migration that affects the first time steps too. Stabilization may take as long as 10 to 30 regular time steps on 8,192 cores and will thus affect performance of efficiency tests that last for a few time steps only, but not of production runs that execute $10^{4}$ time steps or more.

From Figure 12, we observe that weak scaling efficiency stays at $92 \%$ and $88 \%$, respectively, at 8,192 cores. A breakup into components (Figure 13) for both scenarios shows that all components scale sufficiently well. The porous media flow scenario shows some fluctuations in the plot, due to varying numbers of linear solver steps. As the problem size increases, this number is not constant. Therefore, good scaling is given for up to 8,000 cores, which is enough for the purpose of $2 \mathrm{D}$ simulation. 

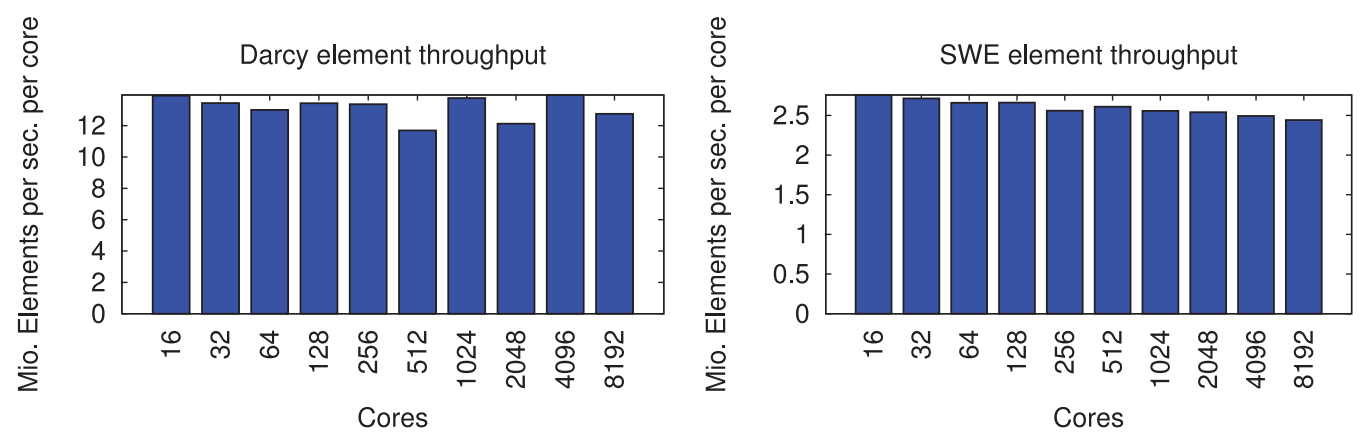

Fig. 12. MPI weak scaling of a dynamically adaptive simulation with up to 8,192 cores and 10G elements on the SuperMUC thin nodes for porous media flow (left) and tsunami wave propagation (right). Porous media flow performance oscillates due to varying numbers of CG iterations per time step in a weak scaling setting. The porous media scenario used seven refinement depth levels with a maximum depth of 40; tsunami wave propagation used at most 31 depth levels with a maximum depth of 39 .
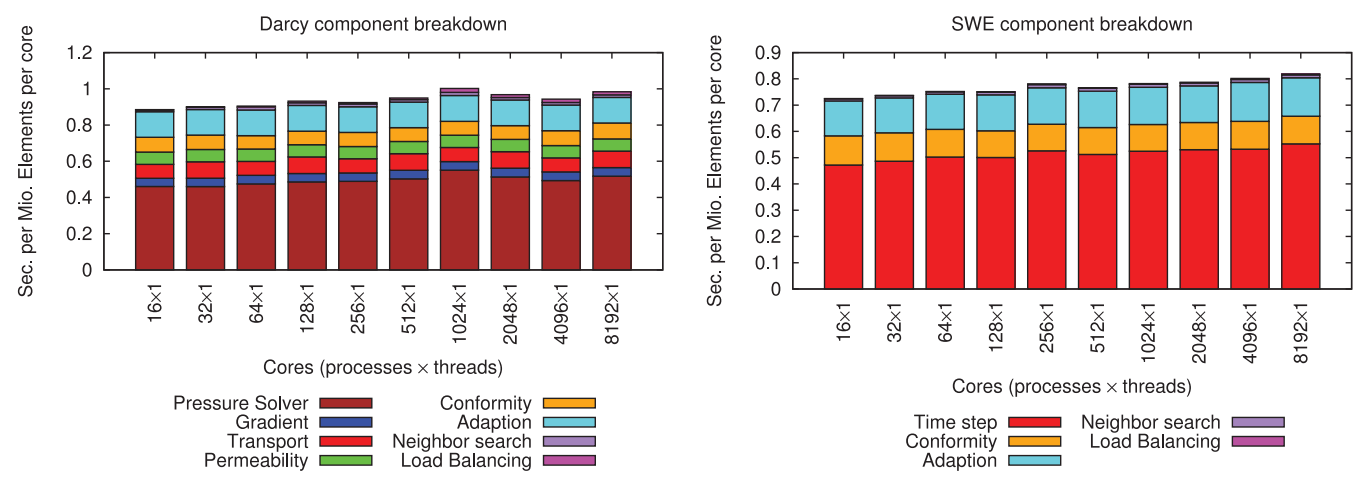

Fig. 13. Component analysis of an MPI weak scaling study for porous media flow (left) and tsunami wave propagation (right). Each bar shows the wall-clock time per simulation time step per core for $1 \mathrm{M}$ elements and split by components. This is not the actual number of elements per core, which depends on adaptive refinement and coarsening, but a normalized value chosen for comparison.

\section{CONCLUSION}

$\operatorname{sam}(\text { oa })^{2}$ is a flexible framework for a dynamically adaptive, parallel solution of PDEs on tree-structured triangular meshes. OpenMP, MPI, and hybrid parallelization are supported, and good strong scaling with around 90\% efficiency is achieved from 16 to 512 cores of SuperMUC. In our weak scaling tests, $\operatorname{sam}(\mathrm{oa})^{2}$ successfully manages up to 10 billion elements on over 8,000 cores with a parallel efficiency of $92 \%$. The stack\&stream approach ensures memory and cache efficiency and offers inherent heuristics for communication and load balancing that scale to large numbers of cores.

With its generic interface designed for $\mathrm{FE}, \mathrm{FV}$, and $\mathrm{DG}$ discretization, $\operatorname{sam}(\mathrm{oa})^{2}$ supports multiple applications, which we demonstrated on test scenarios from twophase porous media flow and tsunami wave propagation. Application-defined kernels for adaptivity allow complex refinement strategies.

While we focus on $2 \mathrm{D}$ mesh refinement, $3 \mathrm{D}$ applications are possible by storing vertical columns of DoFs in each element, thus using triangular prisms as grid cells. Following such a "2.5D" approach, we recently implemented a more complex porous media flow scenario to simulate the 3D SPE10 benchmark [Meister and Bader 2015]. Further application scenarios include domains with a small vertical extent, such as multilayer shallow water equations [Mandli 2013], or 3D problems, where the 
adaptive refinement is restricted to two dimensions (atmosphere models, e.g., Kalise et al. [2011]).

\section{LICENSE AND AVAILABILITY}

$\operatorname{sam}(\text { oa })^{2}$ is licensed under GPL. Its source code is available at https://github.com/ meistero/samoa/.

\section{ACKNOWLEDGMENTS}

We would like to thank Rainer Helmig and Bernd Flemisch (University of Stuttgart, Germany) for helping us with their expertise in modeling and simulation of porous media flow. This work was supported by the Volkswagen Foundation as part of the ASCETE project (http://www.ascete.de/).

\section{REFERENCES}

M. S. Alnæs, A. Logg, K. B. Ølgaard, M. E. Rognes, and G. N. Wells. 2014. Unified form language: A domainspecific language for weak formulations of partial differential equations. ACM Trans. Math. Softw. 40, 2, Article 9 (March 2014), 37 pages. DOI : http://dx.doi.org/10.1145/2566630

M. Bader, C. Böck, J. Schwaiger, and C. Vigh. 2010. Dynamically adaptive simulations with minimal memory requirement-Solving the shallow water equations using Sierpinski curves. SIAM J. Scientific Computing 32, 1 (2010), 212-228. DOI : http://dx.doi.org/10.1137/080728871

M. Bader, A. Breuer, W. Hölzl, and S. Rettenberger. 2014. Vectorization of an augmented Riemann solver for the shallow water equations. In Proceedings of the 2014 International Conference on High Performance Computing and Simulation (HPCS'14). IEEE, 193-201.

M. Bader, K. Rahnema, and C. Vigh. 2011. Memory-efficient Sierpinski-order traversals on dynamically adaptive, recursively structured triangular grids. In Para 2010: State of the Art in Scientific and Parallel Computing, K. Jónasson (Ed.), Vol. 7134. Springer, Berlin, 302-312. DOI:http://dx.doi.org/ 10.1007/978-3-642-28145-7_30

M. Bader, S. Schraufstetter, C. A. Vigh, and J. Behrens. 2008. Memory efficient adaptive mesh generation and implementation of multigrid algorithms using Sierpinski curves. Int. J. Comput. Sci. Eng. 4, 1 (2008), 12-21. DOI : http://dx.doi.org/10.1504/IJCSE.2008.021108

P. Bastian, M. Blatt, A. Dedner, C. Engwer, R. Klöfkorn, M. Ohlberger, and O. Sander. 2008. A generic grid interface for parallel and adaptive scientific computing. Part I: Abstract framework. Computing 82, 2-3 (2008), 103-119. DOI : http://dx.doi.org/10.1007/s00607-008-0003-x

J. Behrens and J. Zimmermann. 2000. Parallelizing an unstructured grid generator with a spacefilling curve approach. In Euro-Par 2000 Parallel Processing (Lecture Notes in Computer Science), A. Bode, T. Ludwig, W. Karl, and R. Wismüller (Eds.), Vol. 1900. Springer, Berlin, 815-823. DOI : http://dx.doi.org/10.1007/3-540-44520-X_112

P. Binning and M. A. Celia. 1999. Practical implementation of the fractional flow approach to multi-phase flow simulation. Adv. Water Resources 22, 5 (1999), 461-478. DOI:http://dx.doi.org/ 10.1016/S0309-1708(98)00022-0

H.-J. Bungartz, M. Mehl, T. Neckel, and T. Weinzierl. 2010. The PDE framework Peano applied to fluid dynamics: An efficient implementation of a parallel multiscale fluid dynamics solver on octree-like adaptive Cartesian grids. Comput. Mechanics 46, 1 (2010), 103-114. DOI:http://dx.doi.org/ 10.1007/s00466-009-0436-x

C. Burstedde, D. Calhoun, K. T. Mandli, and A. R. Terrel. 2013. ForestClaw: Hybrid forest-of-octrees AMR for hyperbolic conservation laws. In Parallel Computing - Accelerating Computational Science and Engineering (CSE'13) (Advances in Parallel Computing), Vol. 25. IOS Press, 253-262. DOI : http://dx.doi.org/ 10.3233/978-1-61499-381-0-253

C. Burstedde, L. C. Wilcox, and O. Ghattas. 2011. p4est: Scalable algorithms for parallel adaptive mesh refinement on forests of octrees. SIAM J. Sci. Comput. 33, 3 (2011), 1103-1133. DOI:http://dx.doi.org/ $10.1137 / 100791634$

C. C. Chueh, M. Secanell, W. Bangerth, and N. Djilali. 2010. Multi-level adaptive simulation of transient two-phase flow in heterogeneous porous media. Comput. Fluids 39, 9 (2010), 1585-1596. DOI : http://dx.doi.org/ 10.1016/j.compfluid.2010.05.011

P. Galvez, J.-P. Ampuero, L. A. Dalguer, S. Somala, and T. Nissen-Meyer. 2013. Dynamic earthquake rupture modeled with an unstructured 3d spectral element method applied to the 2011 M9 Tohoku earthquake. Geophys. J. Int. 198, 2 (December 2013), 1222-1240. DOI : http://dx.doi.org/10.1093/gji/ggu203 
D. L. George. 2008. Augmented Riemann solvers for the shallow water equations over variable topography with steady states and inundation. J. Comput. Phys. 227, 6 (2008), 3089-3113. DOI : http://dx.doi.org/10.1016/j.jcp.2007.10.027

J. Horrillo, Z. Kowalik, and Y. Shigihara. 2006. Wave dispersion study in the indian ocean-tsunami of December 26, 2004. Marine Geodesy 29, 3 (2006), 149-166. DOI : http://dx.doi.org/10.1080/01490410600939140

D. Kalise, I. Lie, and E. F. Toro. 2011. High-order finite volume schemes for layered atmospheric models. CoRR abs/1110.6834 (2011).

R. J. LeVeque. 2002. Finite Volume Methods for Hyperbolic Problems. Cambridge University Press.

R. J. LeVeque, D. L. George, and M. J. Berger. 2011. Tsunami modelling with adaptively refined finite volume methods. Acta Numer. 20 (5 2011), 211-289. DOI : http://dx.doi.org/10.1017/S0962492911000043

A. Logg and G. N. Wells. 2010. DOLFIN: Automated finite element computing. ACM Trans. Math. Softw. 37, 2, Article 20 (April 2010), 28 pages. DOI : http://dx.doi.org/10.1145/1731022.1731030

K. T. Mandli. 2013. A numerical method for the two layer shallow water equations with dry states. Ocean Modelling 72, (2013), 80-91. DOI : http://dx.doi.org/10.1016/j.ocemod.2013.08.001

J. D. McCalpin. 1995. Memory bandwidth and machine balance in current high performance computers. IEEE Computer Society Technical Committee on Computer Architecture (TCCA) Newsletter (Dec. 1995), 19-25.

O. Meister and M. Bader. 2015. 2D adaptivity for 3d problems: Parallel SPE10 reservoir simulation on dynamically adaptive prism grids. J. Comput. Sci. 9, (May 2015), 101-106.

O. Meister, K. Rahnema, and M. Bader. 2012. A software concept for cache-efficient simulation on dynamically adaptive structured triangular grids. In Applications, Tools and Techniques on the Road to Exascale Computing (Advances in Parallel Computing), K. De Boschhere, E. H. D'Hollander, G. R. Joubert, D. Padua, and F. Peters (Eds.), Vol. 22. ParCo 2012, IOS Press, 251-260. DOI : http://dx.doi.org/10.3233/978-1-61499-041-3-251

G. Meurant. 1987. Multitasking the conjugate gradient method on the CRAY X-MP/48. Parallel Comput. 5, 3 (1987), 267-280. DOI : http://dx.doi.org/10.1016/0167-8191(87)90037-8

W. F. Mitchell. 1991. Adaptive refinement for arbitrary finite-element spaces with hierarchical bases. J. Comput. Appl. Math. 36 (1991), 65-78.

W. F. Mitchell. 2007. A refinement-tree based partitioning method for dynamic load balancing with adaptively refined grids. J. Parallel Distrib. Comput. 67, 4 (2007), 417-429.

R. Pajarola. 1998. Large scale terrain visualization using the restricted quadtree triangulation. In Proceedings of the Conference on Visualization (VIS'98). IEEE Computer Society Press, Los Alamitos, CA, USA, 19-26.

A. Pinar and C. Aykanat. 2004. Fast optimal load balancing algorithms for 1D partitioning. J. Parallel Distrib. Comput. 64, 8 (2004), 974-996. DOI : http://dx.doi.org/10.1016/j.jpdc.2004.05.003

S. Popinet. 2011. Quadtree-adaptive tsunami modelling. Ocean Dynamics 61, 9 (2011), 1261-1285. DOI : http://dx.doi.org/10.1007/s10236-011-0438-z

S. Rettenberger. 2012. Ein Paralleler Server Für Adaptive Geoinformation in Strömungssimulationen. Master's thesis. Institut für Informatik, Technische Universität München.

Y. Saad. 1985. Practical use of polynomial preconditionings for the conjugate gradient method. SIAM J. Sci. Statist. Comput. 6, 4 (1985), 865-881. DOI : http://dx.doi.org/10.1137/0906059

K. Schloegel, G. Karypis, V. Kumar, R. Biswas, and L. Oliker. 1998. A performance study of diffusive vs. remapped load-balancing schemes. In ISCA 11th International Conference on Parallel and Distributed Computing Systems. International Society for Computers and Their Applications, 59-66.

M. Schreiber, H.-J. Bungartz, and M. Bader. 2012. Shared memory parallelization of fully-adaptive simulations using a dynamic tree-split and -join approach. In IEEE International Conference on High Performance Computing (HiPC'12), IEEE Xplore, 1-10. DOI : http://dx.doi.org/10.1109/HiPC.2012.6507479

J. R. Shewchuk. 1994. An Introduction to the Conjugate Gradient Method Without the Agonizing Pain. Technical Report. Carnegie Mellon University, Pittsburgh, PA.

L. Velho, L. de Figueiredo, and J. Gomes. 1999. Hierarchical generalized triangle strips. Vis. Comput. 15, 1 (1999), 21-35. DOI : http://dx.doi.org/10.1007/s003710050160

C. A. Vigh. 2012. Parallel Simulation of the Shallow Water Equations on Structured Dynamically Adaptive Triangular Grids. Dissertation. Technische Universitt München, München.

T. Weinzierl and M. Mehl. 2011. Peano - A traversal and storage scheme for octree-like adaptive Cartesian multiscale grids. SIAM J. Sci. Comput. 33, 5 (2011), 2732-2760. DOI : http://dx.doi.org/10.1137/100799071

T. Weinzierl, R. Wittmann, K. Unterweger, M. Bader, A. Breuer, and S. Rettenberger. 2013. Hardware-aware block size tailoring on adaptive spacetree grids for shallow water waves. In 1st International Workshop on High-Performance Stencil Computations (HiStencils'14). HiStencils, 57-64. 
S. Williams, A. Waterman, and D. Patterson. 2009. Roofline: An insightful visual performance model for multicore architectures. Commun. ACM 52, 4 (April 2009), 65-76. DOI :http://dx.doi.org/k10.1145/ 1498765.1498785

G. Zumbusch. 2001. On the quality of space-filling curve induced partitions. Zeitschrift für Angewandte Mathematik und Mechanik 81, Suppl. 1 (2001), 25-28. DOI : http://dx.doi.org/10.1007/3-540-47789-6_4

G. Zumbusch. 2002. Load balancing for adaptively refined grids. Proc. Appl. Math. Mechanics 1 (2002), $534-537$.

Received September 2014; revised December 2015; accepted May 2016 